\title{
Cargo Revenue Management for Space Logistics
}

\author{
by \\ Nii A. Armar \\ B.S., Aerospace Engineering with Information Technology \\ Massachusetts Institute of Technology, 2006 \\ Submitted to the Department of Aeronautics and Astronautics \\ in partial fulfillment of the requirements for the degree of \\ Master of Science in Aeronautics and Astronautics \\ at the \\ MASSACHUSETTS INSTITUTE OF TECHNOLOGY
}

February 2009

(c) Massachusetts Institute of Technology 2009. All rights reserved.

Author

Department of Aeronautics and Astronautics

November 10, 2008

Certified by .

Olivier L. de Weck

Professor of Aeronautics and Astronautics and Engineering Systems

Thesis Supervisor

Accepted by

David L. Darmofal

Chairman, Department Committee on Graduate Students 


\title{
Cargo Revenue Management for Space Logistics
}

by

\author{
Nii A. Armar \\ Submitted to the Department of Aeronautics and Astronautics \\ on November 10, 2008, in partial fulfillment of the \\ requirements for the degree of \\ Master of Science in Aeronautics and Astronautics
}

\begin{abstract}
This thesis covers the development of a framework for the application of revenue management, specifically capacity control, to space logistics for use in the optimization of mission cargo allocations, which in turn affect duration, infrastructure availability, and forward logistics. Two capacity control algorithms were developed; the first is based on partitioning of Monte Carlo samples while the second is based on bid-pricing with high-frequency price adjustments. The algorithms were implemented in Java as a plugin module to SpaceNet 2.0, an existing integrated modeling and simulation tool for space logistics. The module was tested on a lunar exploration concept which emphasizes global exploration of the Moon using mobile infrastructure. Results suggest that revenue management produces better capacity allocations in shorter duration missions, while producing nominal capacity allocations (i.e. those in the deterministic case) in the long run.
\end{abstract}

Thesis Supervisor: Olivier L. de Weck

Title: Professor of Aeronautics and Astronautics and Engineering Systems 


\section{Acknowledgments}

I would like to thank my advisor, Professor Olivier de Weck, from whom I've learned a tremendous amount over the last 2 years. From his first email where he insisted that I call him Oli, he has always made me feel comfortable and a part of the team.

I would also like to thank the whole SpaceNet/ISCMLA team whose hard work and dedication before and during my Masters program has made this thesis possible. I would like to acknowledge Gene Lee, Dr. Robert Shishko, Elizabeth Jordan, and Brian Bairstow of JPL, Erica Gralla, Jaemyung Ahn, Afreen Siddiqi, Matt Silver, Jason Mellein, Christoph Meier, Ariane Chepko, Fabrice Granzotto, and Paul Grogan of MIT, Joe Parrish of PSI, and Dr. Martin Steele, our COTR at NASA KSC.

The first part of my research was funded under a fellowship from the MIT Aeronautics and Astronautics Department, for which I am eternally grateful. The remainder of my research was funded under the NASA Constellation University Institute Program (CUIP). For his excellent oversight of the CUIP project, I would like to say thanks to Tim Propp at NASA JSC.

I would also like to thank all of my office mates on the "wall side" of 33-409. I shared many a laugh and YouTube moment with Wilfried, Maokai, Arthur, Bill, Ryan, SeungBum, and my longtime desk neighbor Gergana.

I would like to extend special thanks to Professor Wesley Harris, who served as my undergraduate advisor and was a great mentor to me throughout my MIT career.

I would also like to thank the whole Aero-Astro department. From day one I have felt welcome and supported, and I simply could not imagine having spent the last 6 years anywhere else. Special thanks goes to Marie Stuppard and Barbara Lechner for their indispensable help and guidance to me throughout my MIT career. I am sure that I speak for many students when I say that the student experience would not be the same without them. In addition I would like to thank Todd Billings, Dave Robertson, and Dick Perdichizzi in the lab for their patience and support as I learned how to build things without burning down the lab (it only took me 4 years).

I would like to thank my roommate and good friend Gaston, who shared the full Aero-Astro experience with me, and ensured that I always had company when coding at $3 \mathrm{AM}$. I would also like to thank the members of my 2007 World Champion DBF team. Adam, Carl, Fu, Brandon, David, Ryan, and George combined to provide the best team experience I have ever had, and probably ever will have.

Finally, I would like to thank my family for their support throughout my life, and especially my 6 years at MIT. 


\section{Contents}

1 Introduction $\quad 17$

1.1 Motivation ............................ 17

1.2 Space Exploration and Logistics . . . . . . . . . . . . . . . . . . . . . . . . . . 17

1.2.1 Mission Types ....................... 17

1.2 .2 Logistics Strategies . . . . . . . . . . . . . . . . . . . . . . 19

1.2 .3 The ISCMLA Framework . . . . . . . . . . . . . . . . . . . . . 19

1.3 Revenue Management . . . . . . . . . . . . . . . . . . . . . . . . . . . . 20

1.3.1 Requirements and Characteristic Features . . . . . . . . . . . . . 20

1.3.2 Industry Examples . . . . . . . . . . . . . . . . . . . . . . 22

1.4 Space Exploration and Revenue Management . . . . . . . . . . . . . . . . . . . . . 23

1.4.1 Suitability for Revenue Management . . . . . . . . . . . . . . . . . . 23

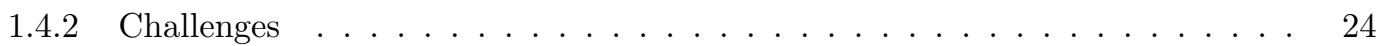

1.5 Thesis Organization . . . . . . . . . . . . . . . . . . . . . . . 24

2 SpaceNet 2.0: Integrated Modeling and Simulation 27

2.1 Scenario Building . . . . . . . . . . . . . . . . . . . . . . . 27

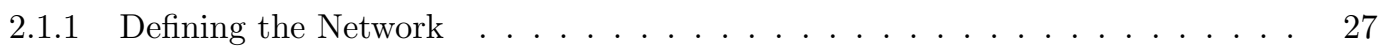

2.1.2 Defining the Mission . . . . . . . . . . . . . . . . . . . 29

2.1.3 Defining Resource Models . . . . . . . . . . . . . . . . . . 30

2.1.4 Forecasting Demand . . . . . . . . . . . . . . . . . . 31

2.1.5 Manifesting Demand . . . . . . . . . . . . . . . . . 32

2.2 Simulation ............................. 32

2.2 .1 Discrete Events . . . . . . . . . . . . . . . . . . . . . 32

2.2.2 Clock Management . . . . . . . . . . . . . . . . . . . 33

2.2.3 System State and Histories . . . . . . . . . . . . . . . . . 33

2.2 .4 Performance Measures . . . . . . . . . . . . . . . . . . . . 35

2.3 Data Management . . . . . . . . . . . . . . . . . . . . . . . . . 35

2.4 Chapter Summary . . . . . . . . . . . . . . . . . . . . 36

3 Revenue Management $\quad 37$

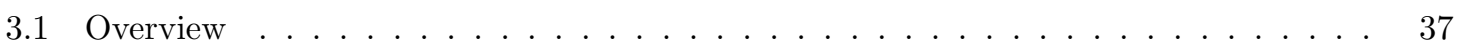

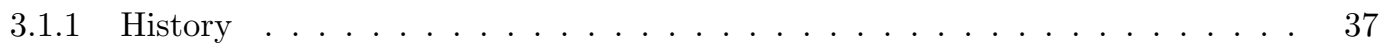

3.1 .2 Airlines .......................... 37

3.1 .3 Hotels \& Rental Cars . . . . . . . . . . . . . . . . . . . . 38

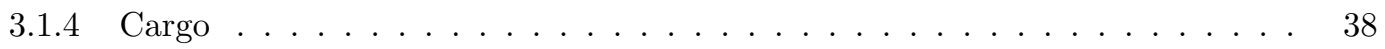


3.1 .5 Other Industries . . . . . . . . . . . . . . . . . . . . . . . . . . 39

3.2 Revenue Management Disciplines . . . . . . . . . . . . . . . . . . . . . . . 39

$3.2 .1 \quad$ Forecasting and Estimation $\ldots \ldots \ldots \ldots \ldots$

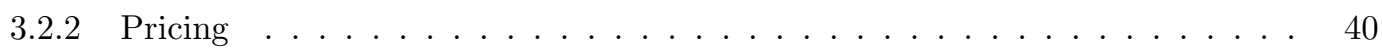

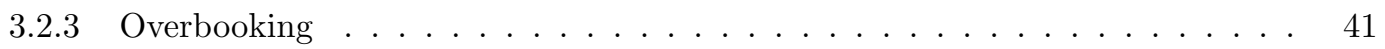

3.3 Capacity Control . . . . . . . . . . . . . . . . . . . . . . . 41

3.3 .1 Levels of Decision Making . . . . . . . . . . . . . . . . . . . . . . . . . . 42

3.3 .2 Littlewood's Rule . . . . . . . . . . . . . . . . . . . . . . . 43

3.3.3 Expected Marginal Seat Revenue . . . . . . . . . . . . . . . . . . . . . . . 44

3.3 .4 Dynamic Controls . . . . . . . . . . . . . . . . . . . . . 44

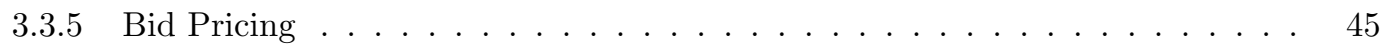

3.4 Implementation . . . . . . . . . . . . . . . . . . . . . 45

3.5 Chapter Summary . . . . . . . . . . . . . . . . . . . . 46

4 SpaceNet Revenue Management $\quad 47$

4.1 Conceptual Framework . . . . . . . . . . . . . . . . . . . . . . 47

4.1 .1 Exploration Value . . . . . . . . . . . . . . . . . . 47

4.1 .2 Marginal Exploration Value . . . . . . . . . . . . . . . . . . . . . 49

4.1 .3 Relative Exploration Value . . . . . . . . . . . . . . . . . 50

4.1.4 Defining the Available Resource . . . . . . . . . . . . . . . . 50

4.2 EMEV Algorithm . . . . . . . . . . . . . . . . . . . . . 51

$4.2 .1 \quad$ Estimating Prices . . . . . . . . . . . . . . . . . . . 51

4.2 .2 Generating Demands and Partial Sums _. . . . . . . . . . . . . . 52

$4.2 .3 \quad$ Finding Optimal Protection Levels . . . . . . . . . . . . . . . . 53

4.3 Greedy Bid-Price Algorithm . . . . . . . . . . . . . . . . . . . 55

4.4 SpaceNet RM Implemenation . . . . . . . . . . . . . . . . . . . 55

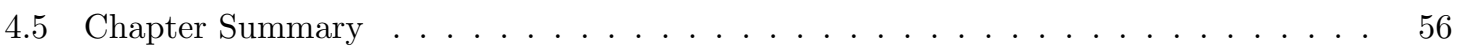

5 Results and Conclusions $\quad 59$

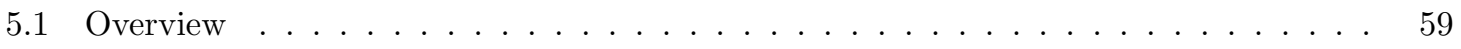

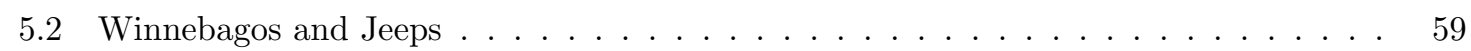

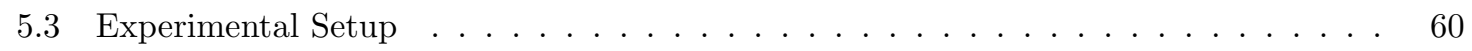

5.4 Experimental Results . . . . . . . . . . . . . . . . . . . . . 62

5.4 .1 Mission Duration . . . . . . . . . . . . . . . . . . . 62

5.4 .2 Spares Uncertainty . . . . . . . . . . . . . . . . . . . . . . 62

5.4 .3 Mobile Outpost Protection Levels _. . . . . . . . . . . . . . . . 64

5.5 Algorithm Comparison . . . . . . . . . . . . . . . . . . . 65

6 Conclusions $\quad 67$

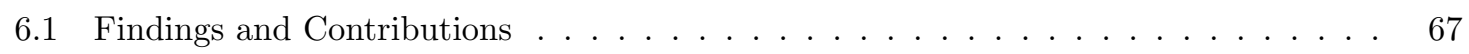

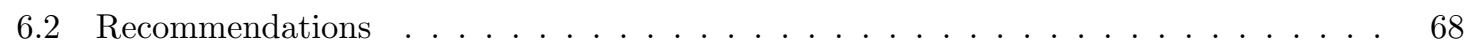

6.3 Future Work . . . . . . . . . . . . . . . . . . . . . . 69

$\begin{array}{ll}\text { A Network Representation } & \mathbf{7 1}\end{array}$

A.1 The Static Network . . . . . . . . . . . . . . . . . . . . . 71

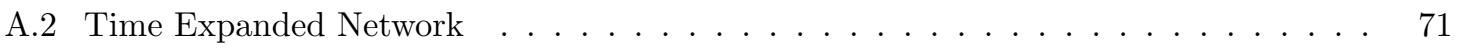


B Measures of Effectiveness $\quad 73$

B.1 Basic Logistics Performance . . . . . . . . . . . . . . . . . . . . . . . . 73

B.1.1 Crew Surface Days $($ CSD $)[$ crew-days $] \ldots \ldots$. . . . . . . . . . . 73

B.1.2 Exploration Mass Delivered (EMD) $[\mathrm{kg}] \ldots \ldots \ldots \ldots$. . . . . . . . . . . . . . . . . . . . 73

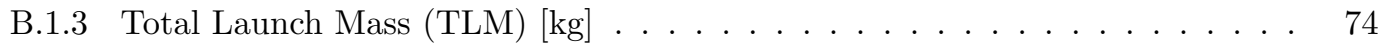

B.1.4 Up-mass Capacity Utilization (UCU) $[0,1] \ldots \ldots \ldots \ldots \ldots . \ldots \ldots$

B.1.5 Return-mass Capacity Utilization (RCU) $[0,1] \ldots \ldots \ldots \ldots \ldots$. . . . . . . . . . . . . 75

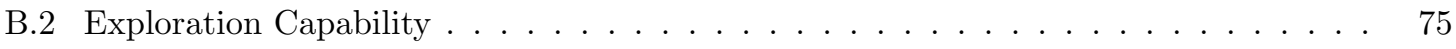

B.2.1 Exploration Capability $[$ crew-day-kg] . . . . . . . . . . . . . . . 75

B.2.2 Relative Exploration Capability . . . . . . . . . . . . . . . . . . . . 76

$\begin{array}{ll}\text { C ATHLETE } & 77\end{array}$ 


\section{List of Figures}

1-1 Simulated logistics strategy for nominal Constellation campaign . . . . . . . . . . . 21

1-2 Functional classes of supply for human space exploration. . . . . . . . . . . . . . 21

$1-3$ Thesis roadmap . . . . . . . . . . . . . . . . . . . . . 25

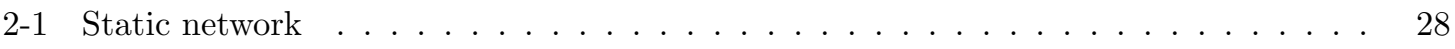

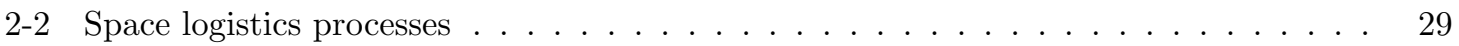

2-3 SpaceNet 2.0 variable time advance clock management $\ldots \ldots \ldots \ldots \ldots$

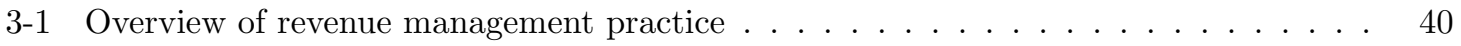

$3-2 \quad$ Typical airline revenue management system . . . . . . . . . . . . . . . . 46

$4-1 \quad$ System availability estimation process $\ldots \ldots \ldots \ldots \ldots$

4-2 Availability-versus-resource curve for a nominal ORU . . . . . . . . . . . . . 50

4-3 Available cargo resource for application of revenue management . . . . . . . . . . 51

4-4 Demand vector $\left(d_{k}\right)$ and partial sum vector $\left(S_{k}\right)$ generation - general case $\ldots \ldots . \quad 54$

4-5 Demand vector $\left(d_{k}\right)$ and partial sum vector $\left(S_{k}\right)$ generation - quantitative example

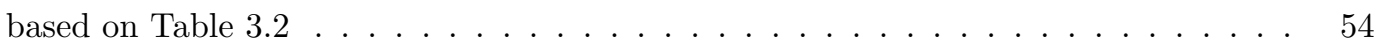

4-6 Plot of the partial sums S1, S2 and the resulting Monte Carlo estimates of optimal protection levels for 25 simulated data points . . . . . . . . . . . 55

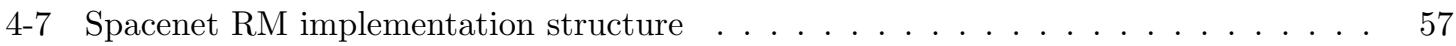

5-1 Winnebagos and Jeeps mobile outpost exploration concept . . . . . . . . . . . . 61

5-2 Normalized MEVs versus mission duration using EMEV algorithm . . . . . . . . 63

5-3 Normalized MEVs versus mission duration using Greedy-Bid Price algorithm . . . . 63

5-4 Evolution of MEV versus K-factor as a fraction of the deterministic case estimate . . 64

5-5 Mission-by-mission capacity allocations for Winnebagos and Jeeps mobile outpost scenario using the EMEV algorithm . . . . . . . . . . . . . . 66

5-6 Mission-by-mission capacity allocations for Winnebagos and Jeeps mobile outpost scenario using the Greedy Bid-Price algorithm . . . . . . . . . . . . . 66

A-1 An Earth-Moon time expanded network . . . . . . . . . . . . . . . . . . 72

C-1 All-Terrain Hex-Legged Extra-Terrestrial Explorer (ATHLETE) robotic vehicle . . . 77 


\section{List of Tables}

2.1 Resource Models and Classes of Supply _ . . . . . . . . . . . . . . . . . . 30

3.1 Basic demand forecast with prices, two-class example . . . . . . . . . . . . . 40

3.2 Basic demand forecast with prices, three-class example . . . . . . . . . . . . . . . 44

5.1 Winnebagos and Jeeps mobile outpost exploration detailed plan . . . . . . . . . . . 60

5.2 Demand model for Winnebagos and Jeeps mobile outpost scenario . . . . . . . . . . 62 


\title{
Nomenclature
}

\author{
$C$ maximum allocable amount of a resource \\ $b_{j}$ booking limit for class $\mathrm{j}$ \\ $y_{j}$ protection level for class $\mathrm{j}$ \\ $V(x)$ optimal expected revenue given unused resource amount $x$ \\ $\Delta V$ Delta- $\mathrm{V}$
}

CoS Class of Supply

CEV Crew Exploration Vehicle (Orion)

CTB Cargo Transfer Bag

CWC Crew Water Container

DRM Design Reference Mission

EC Exploration Capability

EMEV Expected Marginal Exploration Value

EMSR Expected Marginal Seat Revenue

ESAS Exploration Systems Architecture Study

EV Exploration Value

EVA Extra-vehicular Activity

FM Forecasting Module

ISCMLA Interplanetary Supply Chain Management

ISCMLA Interplanetary Supply Chain Management and Logistics Architectures

ISS International Space Station

IVA Intra-vehicular Activity

LEO Low Earth Orbit

LEO Low Lunar Orbit 
LL-D Lunar Lander - Descent Stage

MBTF Mean Time Between Failure

MEC Marginal Exploration Capability

MEV Marginal Exploration Value

MOE Measure of Effectiveness

ORU Orbital Replacement Unit

PLM Pressurized Logistics Module

REC Relative Exploration Capability

REV Relative Exploration Value

RM Revenue Management

RPM Resource Pricing Module

SHOSS SPACEHAB Oceaneering Space Systems

SRU Shop Replacement Unit

TEN Time Expanded Network

TPM Technical Performance Measure 


\section{Chapter 1}

\section{Introduction}

\subsection{Motivation}

Few things capture the imagination as does the exploration of space. However, space exploration is inherently expensive and complex and thus missions are infrequent. There is a huge opportunity cost associated with each and every kilogram of mass launched into orbit and it is critical to maximize the value derived from each and every mission. This paper discusses the extension of the Interplanetary Supply Chain Management and Logistics Architectures (ISCMLA) framework to include the use of revenue management (RM), which is the optimization of the revenue or benefit earned from a fixed, perishable resource [1], in the design, modification, and evaluation of space logistics architectures.

\subsection{Space Exploration and Logistics}

The next generation of human space exploration will be about true exploration rather than visitation. The focus is on developing the ability to sustain long-term human human presence while reaching deeper into space than ever before. Space logistics, or the movement, storage, and tracking of all crew and equipment necessary to carry out an exploration mission or campaign [2], aims to tackle the challenge of supporting true exploration. It encompasses nearly every aspect of space flight operations with the exception of vehicle and infrastructure design. The ultimate goal is to maximize exploration potential given vehicle performance and infrastructure capabilities, a process which involves answering two key questions:

1. How best to ship cargo and supplies given an exploration architecture?

2. What cargo and supplies should be shipped for a given mission profile?

\subsubsection{Mission Types}

\section{Unmanned Cargo}

Unmanned cargo missions, as the name suggests, are missions conducted with uncrewed vehicles for the purpose of transporting necessary cargo from Earth to in-space exploration sites. Usually unmanned cargo missions are used to deliver large infrastructure items such as habitats and power equipment; they are also used to resupply logistics in support of crewed missions. Unmanned cargo 
flights are particularly important at the beginning of exploration campaigns where their large cargo capability is critical in quickly establishing a presence at a site, including an initial inventory. The

International Space Station (ISS) relies on unmanned missions carried out by the Russian Progress vehicle to help meet logistical needs [3].

\section{Sortie}

A sortie is a stand-alone mission of a relatively short duration (typically 2 weeks or less) that allows the exploration of a site without the need to preposition habitats, infrastructure, and consumables. Sortie missions also allow for exploration of high-interest science sites or scouting of future lunar outpost locations. In sorties, crew members typically live in the vehicle they travel in, and have limited mobility around the site. The crew has the capability to perform daily EVAs (extravehicular activity), typically with all crew members involved to maximize the scientific and operational value of the mission. All of the Apollo missions and most of the Space Shuttle missions have been sortie-style missions.

\section{Outpost}

Outpost missions are a series of extended flights to the same location. The longer duration (typically 1-6 months per flight) means that outpost missions usually involve significantly more infrastructure than sorties. The crew usually has a dedicated habitat separate from the transportation vehicle, which in turn drives the need for more advanced power generation, in-situ resource utilization, and cargo storage infrastructure. Outpost missions also tend to include more advanced surface mobility infrastructure to allow for expansive exploration, rather than being limited to the immediate area around the outpost site. While surface operations during sortie missions are typically dominated by EVAs, outposts typically feature a more balanced schedule of EVA and IVA (intravehicular activity) due to limitations from the extreme radiation environment in space, the fatiguing nature of EVA operations, space suit maintenance and repair, and portable life support system logistics [4]. Outposts are not limited to surface locations, but can also include in space sites. The International Space Station (ISS) is an outpost in low earth orbit, and outpost missions to ISS are called "expeditions".

\section{Mobile Outpost Concept}

The mobile outpost is a hybrid of the sortie and outpost mission types. Mobile outposts combine some of the best aspects of the sortie and outpost mission types by providing the operational flexibility to explore multiple sites while maintaining more infrastructure in service between missions, thereby increasing exploration capability at each site. However, mobile outposts depend first and foremost on efficient, lightweight mobility systems, and also on highly capable power systems integrated into each lander, large habitat shells that can double as pressurized logistics modules (PLMs), and simplified mating (access, fluids and electronics) of habitat and/or logistics modules. In other words, mobility adds technological and development complexity to an exploration program. To date, no space exploration campaign has relied on the mobile outpost concept, however the increasing capability of mobility platforms like NASA's ATHLETE have brought mobile outposts to the forefront (see Appendix C). 


\subsubsection{Logistics Strategies}

There are several different logistics methodologies which combine with the various mission types to form an exploration architecture. The ISCMLA framework defines four different classifications of logistics methodologies based on the study of terrestrial supply chains:

Pre-positioning This approach focuses on sending cargo and supplies to the exploration site ahead of the crew, or more generally when the demand materializes [5]. It is typically achieved through the use of unmanned cargo flights early in a campaign that deliver supplies and infrastructure in bulk. However, pre-positioning can also be achieved by using excess cargo capacity on manned flights to build up a gradual stock of supplies.

Carry-along In this approach, all cargo needed for a given mission is brought along on that mission; in essence each mission is self-contained, and its duration and scope is limited by the cargocarrying capability of the vehicles used for that mission.

Resupply In this approach, some cargo is carried along by the crew, but is then supplemented by scheduled and/or need-based resupply flights. Scheduled resupply flights are pre-determined cargo deliveries to meet predicted demands (based on historical demand models). In practice, need-based resupply flights are difficult to achieve due to the long lead time required for launch preparation.

Depot This approach involves the use of an intermediate distribution center somewhere between the origin and destination of cargo items. The depot methodology stems from the terrestrial concept of a multi-echelon supply chain where inventory is held in a depot until it is demanded by the retailers or customers.

Conceptually, mission types and logistic methodologies are decoupled. However, in practice, certain logistics methodologies work much better with certain mission types. For example, although it is possible to have a "super sortie" in which an unmanned cargo flight supports a future crewed flight, sorties almost always employ a carry along strategy. As exploration architectures become more advanced, they are supported by increasingly advanced interplanetary supply chains utilizing more advanced logistics methodologies. In many cases, these methodologies will be hybrids of the methodologies described above. Figure 1-1 shows a simulated logistics strategy for the Constellation lunar architecture, which also uses a mix of carry-along and pre-positioning [6].

\subsubsection{The ISCMLA Framework}

The ISCMLA project aims to study human space exploration from the logistics perspective. As a result, the modeling framework is quite different from those of traditional space exploration studies. It focuses primarily on the flow of crew and materials through time and space, with compact parametric representations of vehicle form and function. The following terms are commonly referred to in the Interplanetary Supply Chain Management and Logistics Architectures (ISCMLA) framework, which includes the following concepts:

Classes of Supply (CoS) capture the main functional categories of cargo that are needed to support human exploration. Figure 1-2 shows the ten primary classes, which are further divided into forty-four sub-classes and are used for demand estimation and manifesting of individual missions. This detailed CoS definition enables fine-grained modeling of material flows that 
enables analysts to determine not only if enough supplies are at a given site, but also that there are the right types of supplies at the right time.

Resources are the basic unit the producer uses to generate exploration value

Networks are graphs of nodes and edges (i.e. locations and transportation links) that define and connect all points of interest. Networks are also traditionally used in terrestrial transportation systems to represent physical locations and the transportation links of various modes between them. For space transportation networks, the concept of a location is abstracted to represent points of interest, such as orbits or Lagrange points. Note that transportation links in space are generally time-varying due to astrodynamical motion (see Section 2.1.1).

Processes are the building blocks of scenarios ${ }^{1}$. They describe how elements and supplies are allowed to move through the network over time.

Measure of Effectiveness (MOEs) quantitatively evaluate specific space exploration scenarios and interplanetary supply chains. Note that MOEs are only proxy metrics for comparative purposes, not for absolute forecasting.

Technical Performance Measures (TPMs) are feedback metrics that inform the user about infrastructure performance, resource utilization, and safety margins.

\subsection{Revenue Management}

Revenue management (RM) deals with the maximization of the revenue or benefit earned from a fixed, perishable resource in uncertain conditions [1]. While revenue management as a formal practice has its foundations in the airline industry, it has spread in the last two decades to cargo services, hotels, car rental companies, and e-commerce. These industries, particularly airlines and cargo services, have many features that are analogous to those of space exploration. However, at the time of writing, to the author's best knowledge RM has not been used in space logistics. The goal of this thesis is to explore the applicability of RM to space logistics, and the following sections present the space analogy in more detail.

\subsubsection{Requirements and Characteristic Features}

The basic revenue management problem requires four entities: customers, producers, resources, and revenue. Customers seek to attain some perishable resource, which are units of capacity managed by the producer. Each customer has a price they are willing to pay for that resource. The producer controls the allocation of the resource and therefore "produces" resource bundles that are offered to customers. The challenge for the producer is to sell the right resource(s) to the right customer at the right time in order to maximize revenue.

Most transactional business models are candidates for revenue management at some level. However, only in certain types of these businesses do the costs and complexity of implementing a revenue management system outweigh the benefits. Muller defines four characteristics that make a particularly good revenue management problem [7]. These are:

\footnotetext{
${ }^{1} \mathrm{~A}$ scenario is the full specification of the logistical aspects the user wishes to model (see Section 2.1 for more information)
} 


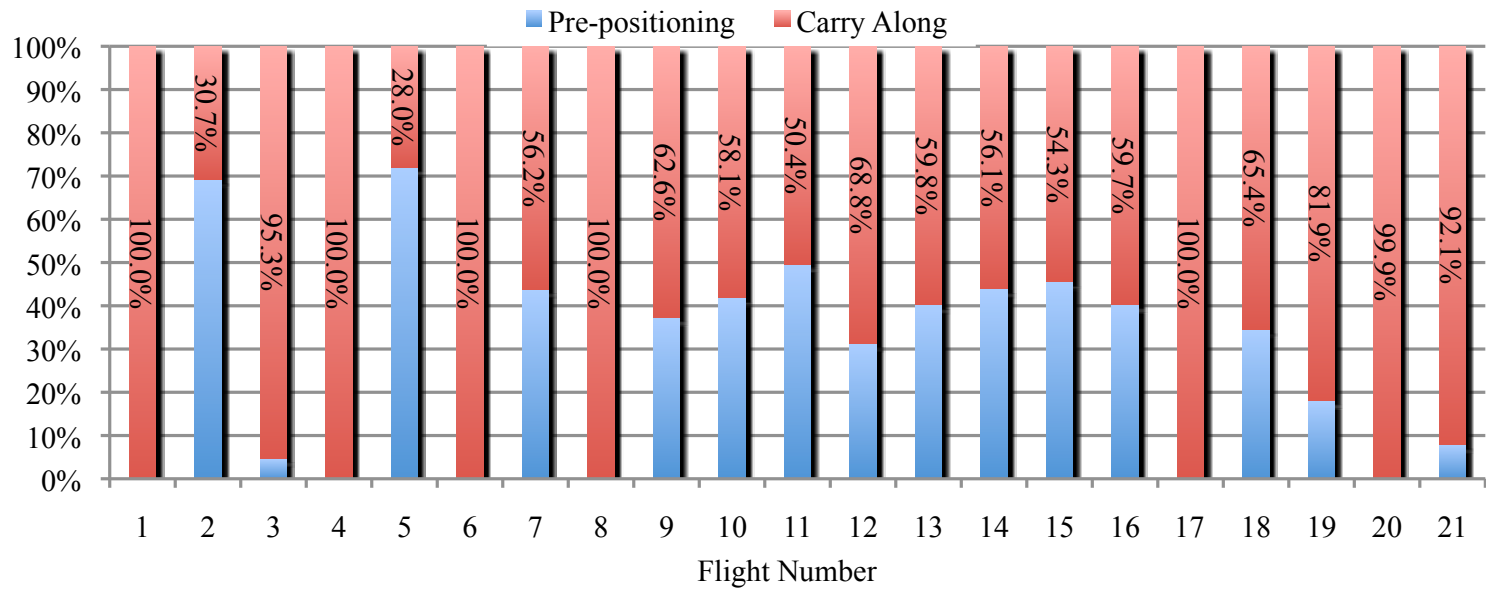

Figure 1-1: Simulated logistics strategy for nominal Constellation campaign. The strategy shown uses a combination of pre-positioning and carry-along to maintain mission robustness through safety stocks (pre-positioning) while allowing for flexibility to adapt mission profiles (carry-along).

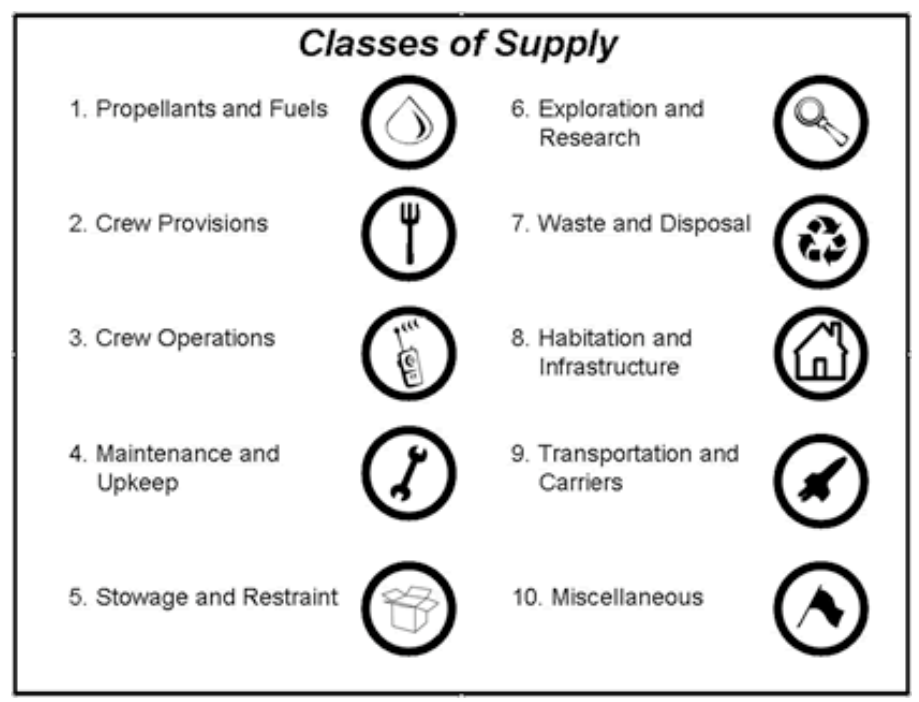

Figure 1-2: Functional classes of supply for human space exploration. 
1. Customer Heterogeneity: customers place different values on resource units. The possibility of segmenting price-sensitive customers is key in implementing revenue management. The most common mechanism used to segment customers is the time of purchase; that is, the less price-sensitive customer generally purchases nearer the time of production.

2. Demand Uncertainty: demand is stochastic, difficult to predict, and cannot be satisfied using existing stocks.

3. Limited operational flexibility: resource units are limited and there is a consistent mismatch between the supply and demand. Additionally, the means to balance supply and demand are limited.

4. Standardized Product Range: the nature of resources on offer remains essentially unaltered over a substantial period of time. This is key to being able to predict future demand.

In other words, A good revenue management candidate industry sells products that are perishable, faces time-varying demand, and is burdened with large fixed costs while variable costs are small in the short run. Due to the low marginal cost and high fixed cost condition, increased revenue essentially translates directly to increased profits.

\subsubsection{Industry Examples}

The airline industry is the prototypical example of the characteristics defined in Section 1.3.1. First, airlines certainly experience customer heterogeneity; business travelers, vacation-goers, students, a host of other groups each have different valuations of the same resource (a seat on the plane). Second, the airlines cannot satisfy demands using existing stocks. It is impossible to consistently satisfy demand for a Friday evening flight from Seattle to Boston with seats on Thursday morning flight. Third, aircraft are large, blocky, and expensive capital investments that have a very finite capacity. It is prohibitively difficult and expensive for airlines to expand or contract their supply in the short or even medium term; they cannot simply make more products (seats) to sell to meet demand. Finally, seats on an aircraft are an extremely standardized product, even across competitors. And the same product is offered whether demanded 30 days or 30 minutes before production (departure).

The initial success of Revenue Management in the airline industry drove an expansion first into the hotel and rental car industries, and then further into cargo shipping, restaurants, and a whole host of other industries. Depending on the needs of the industry, revenue management is used to increase resource utilization (sell more products), segment customers through packaging resources, adjust demand profiles, and/or dynamically price resources.

The following provides a brief overview of how revenue management is used in some key industries:

Hotels use RM techniques to provide special rate packages for periods of low occupancy. They also use overbooking policies (refer to Section 3.2.3) to compensate for cancellations and no-shows.

Restaurants use RM to even out demand over the course of the day. They offer discount coupons or charge reservation fees and higher meal prices during peak periods in order to move customers to off-peak periods.

Attractions (e.g. theme parks and carnivals) set different admission charge levels, provide jointentry tickets, group discounts, coupons, membership rates. 
Rental car companies use RM-based controls to vary prices frequently according to demand, and to accept or reject booking requests. A big driver in this industry is the balance of weekend demand from vacationers and weekday demand from business travelers.

Cargo and freight companies use RM to determine the price of a shipment based on space required, location and comfort. They also use RM to determine the optimal ship size and capacity for each shipment class (e.g. overnight, 2nd day, and ground).

IT Services use RM to allocate administrator time, computing capacity, storage, and network capacity among segments of customers and determine an appropriate price for each segment.

Sections 3.1.2- 3.1.5 present an overview of the unique aspects of RM problems in each of the above industries and related research.

\subsection{Space Exploration and Revenue Management}

Typically, the logistics strategies are developed in response to mission profiles. Within the logistics strategy, cargo is delivered to meet pre-defined availability targets. The use of RM in this process allows this question to be approached from a different perspective. Like in the airline and cargo examples, in space logistics revenue management can be used to allocate cargo delivery capacity (i.e. cargo mass and volume) in order to maximize productive exploration given uncertainty in demand. This in turn defines the availability targets, which helps to define the logistics strategy. Combined with the existing forward approach, this reverse approach allows a bi-directional approach to the design of logistics strategies. When paired with the SpaceNet simulation framework, to be discussed in Chapter 2, revenue management may have the following potential applications:

- Development of design reference missions (DRMs), particularly for exploration architectures that rely on extended sorties or mobile outposts.

- Refining mission profiles in response to vehicle performance and demand uncertainty.

- Optimal manifesting of spacecraft in the operational phase of missions.

\subsubsection{Suitability for Revenue Management}

In the airline case, for example, it is fairly straightforward to identify the customers, producers, resources, and revenue. The customers are passengers, the producers are the airlines, the resources are the seats, and the revenue is money. The space exploration example is perhaps more subtle than the airline example, but nonetheless it has both the requirements and characteristic features to be a strong candidate for the implementation of revenue management. There are essentially there are two types of customers: 1) the exploration agents ${ }^{2}$, and 2) the surface infrastructure at the exploration site. The producers are the various agencies conducting exploration, e.g. NASA or ESA, which must manage their resources in order to maximize their utility from exploration. The resource is the combined mass and volume available for cargo on spacecraft heading to the exploration site; this can be broken down into a pressurized and unpressurized component. Finally, the revenue is the the contribution of the item consuming the resource to productive exploration.

Not only does space exploration have the major entities for the implementation of revenue management, it also shares the characteristic features. These are:

\footnotetext{
${ }^{2}$ Exploration agents can refer to either human crew or robotic systems
} 
- Heterogenous Customers: Exploration agents (generally crew, but sometimes robotic systems) generate demands for provisions and operational equipment. Surface infrastructure are those elements needed to support the exploration agents. These include habitats, power units, and mobility systems and their demand is primarily in terms of spares. These customers demand different amounts of the resource and "pay" different amounts for the resource.

- Demand uncertainty: Demand for crew provisions is relatively deterministic on average, particularly for longer missions. In contrast, demand for spares is extremely uncertain due to limited production quantities for flight hardware, relatively immature designs (compared to most Earth-bound complex systems), and environmental factors.

- Limited Operational Flexibility: Space flights are infrequent, typically scheduled far in advance, and face very tight mass margins. There is very limited ability to change supply to meet demand.

- Standardized Product Range: the contribution of the bundle of manifested resource to productive exploration. Ultimately exploration agencies are in the business of maximizing their benefits from exploration.

\subsubsection{Challenges}

One major challenge in the implementation of RM in space logistics is the definition of revenue. Ultimately exploration agencies are in the business of maximizing their benefits from exploration, as complex as those may be to define or quantify. Benefits can be in the form of scientific discovery, resource discovery, operational knowledge, and/or political gain.

A related challenge is that the revenue is not earned at the time of "sale". Generally speaking, the revenue earned from a customer is not known when a spacecraft is launched, or even when it arrives at the exploration site. Rather revenue is generated on the back end when the item is actually used in the mission. Thus the decision for the producer in this problem is not only one of ensuring that resources are sold to the most high-revenue customers, but also whether or not each customer will actually pay for the resource within a given time horizon.

\subsection{Thesis Organization}

This thesis consists of six chapters, as shown in Figure 1-3. These are:

Chapter 2 gives an introduction to SpaceNet, an integrated modeling and simulation tool. SpaceNet, co-developed by MIT and JPL, allows the user to explore the various logistics strategies discussed Chapter 1. In particular this chapter talks about SpaceNet 2.0, the 2nd generation of the software, which has added flexibility and capability to support more advanced logistical concepts.

Chapter 3 presents foundational revenue management concepts including price-based and quantitybased revenue management. It also provides an overview of various formulations and solution approaches.

Chapter 4 presents the main contribution, the SpaceNet Revenue Management module (SpaceNet $\mathrm{RM}$ ), which is capable of optimizing campaign exploration performance under uncertainty. This chapter also introduces a space logistics-based definition of revenue. 
Chapter 5 presents two case studies where revenue management was applied to help evaluate proposed campaigns.

Chapter 6 outlines several future directions for SpaceNet RM. It concludes with a summary of contributions.

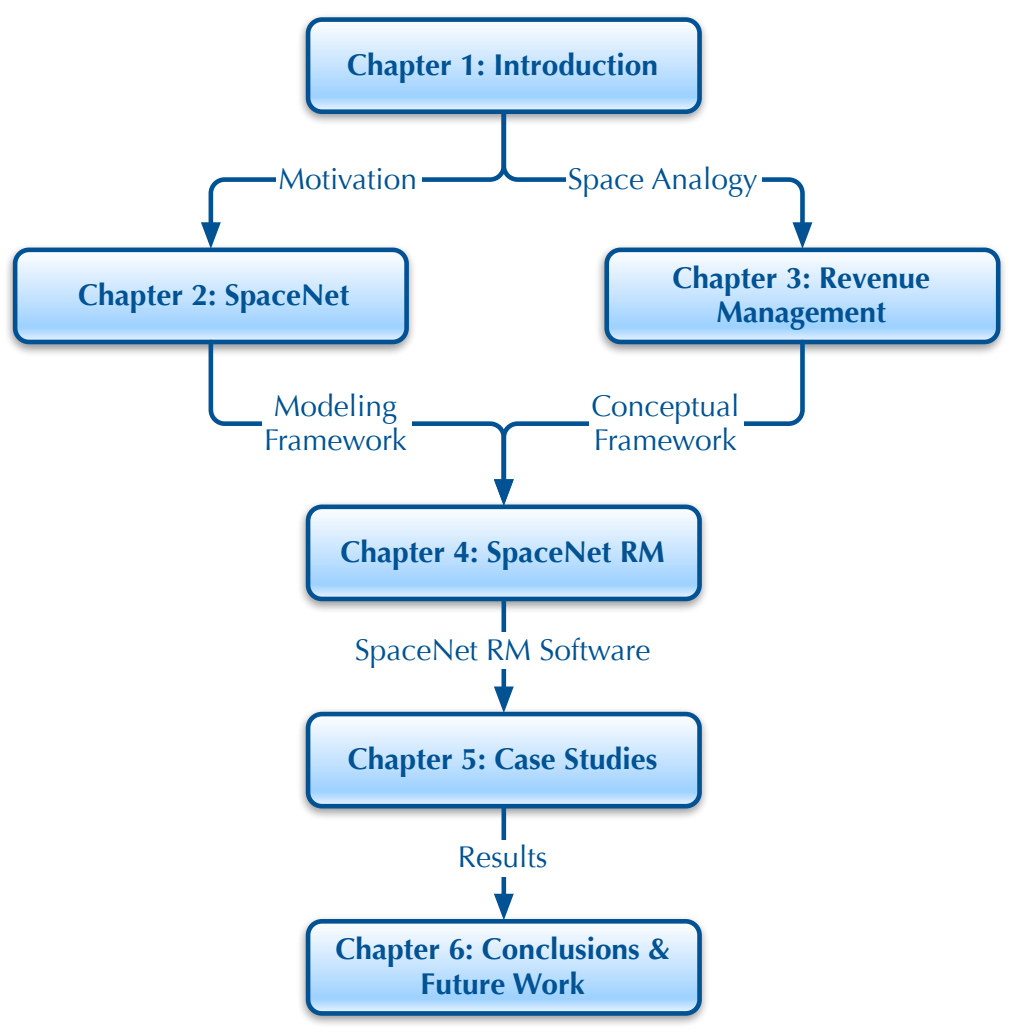

Figure 1-3: Thesis roadmap 


\section{Chapter 2}

\section{SpaceNet 2.0: Integrated Modeling and Simulation}

The goal of SpaceNet is to allow a user to model and evaluate an exploration mission as an interplanetary supply chain with the purpose of answering key logistics questions [8]. These answers provide insight into the bottlenecks in the transportation architecture, strategies to maximize exploration capability, and the impact of uncertainty on logistics strategies. SpaceNet models and simulates processes, element instantiations, and supply item levels, and other logistical aspects of a campaign in a high fidelity, enabling it to respond to complex issues such as campaign robustness and contingency analysis while providing an end-to-end analysis capability.

\subsection{Scenario Building}

A scenario is the full specification of the logistical aspects the user wishes to model. It essentially defines the who, what, when, where, and how of all material flows while appropriately abstracting the how of the technologies that enable space exploration. Building a scenario involves 5 basic steps:

1. Defining a network

2. Defining the mission (i.e. the various exploration and transportation processes)

3. Defining resource models

4. Manifesting supply items (i.e. implementing a logistics strategy)

5. Processing simulation feedback

The following sections provide an overview of each of these steps, and upgrades and added features in SpaceNet 2.0, which is the most recent version of SpaceNet developed at the time of writing. The upgrades to be discussed are designed to allow more flexible modeling, greater interoperability with other tools and models, and to support the implementation of revenue management in SpaceNet.

\subsubsection{Defining the Network}

Unlike most terrestrial networks, space networks have time varying properties; a path that may be available at one time and may be unavailable at another. SpaceNet addresses these properties 
by defining two types of networks. First, there is the static network which represents the set of physical locations (i.e. nodes) and the possible connections between them (i.e. arcs). Second, there is the time-expanded network (TEN) which adds the dimension of time to the static network to enable dynamic behavior, while abstracting the details of orbital mechanics. The time-expanded network has the ability to capture the time-dependency of interplanetary trajectories, including launch windows and tradeoffs between fuel consumption and time-of-flight. Appendix A provides some additional detail about both types of networks and their representation.
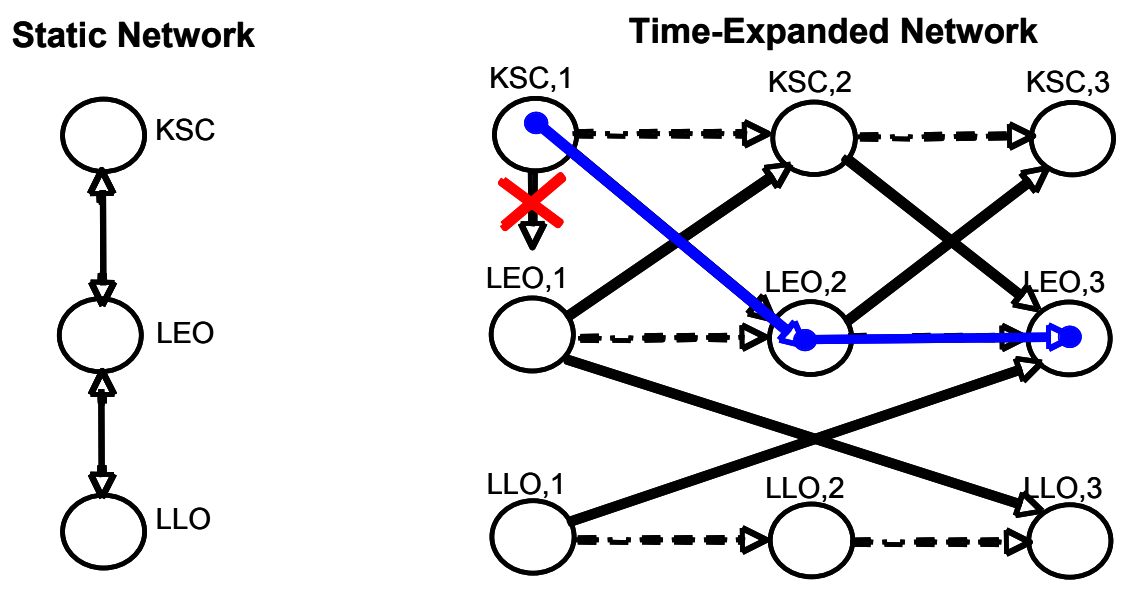

Figure 2-1: Left: Static network including NASA Kennedy Space Center (KSC), Low Earth Orbit (LEO), and Low Lunar Orbit (LLO). Right: the corresponding time expanded network. In this network, one can see that moving from KSC to LEO requires one time step. Therefore it is impossible to move from KSC,1 to LEO,1 while it is possible to move from KSC,1 to LEO,2. It is however, always possible to stay at a node, and therefore a movement from LEO,2 to LEO, 3 is allowed.

To build a network, the user first defines the static network explicitly by adding desired nodes. As a user adds and removes nodes, arcs are automatically added and removed accordingly to show the possible paths throughout the static network in real time. The time expanded network is automatically generated from the user constructed static network based on a chosen time step $\Delta t$. Of course, time expanded networks can grow to be very large depending on the size of the static network and/or the length of the scenario. For example, a time expanded network for a 3-year scenario (about 1000 days) with 10 nodes and a time step of 0.1 days (Earth days) and will have more than 100,000 nodes. This is an issue both in terms of the users ability to manage all the information, and from a computational resource management perspective. To address both issues, the user interacts with the TEN through processes, which identify which parts of the TEN experience logistical activity. Processes, discussed in Section 2.1.2, enable the generation of a "sparse" time expanded network, which address both ease of use and resource management.

SpaceNet 2.0 also features a shortest path algorithm that can assist the user in finding the shortest path between two nodes (if any exists) based on minimizing either cumulative $\Delta V$, duration, or number of orbital transfers (i.e. arcs). The algorithm uses an $A^{*}$ search to look through a database of stored trajectories and returns a pathway, or series of connected arcs to the user. If the pathway contains nodes not in the network at the time of search, the software gives the user the option to add them into the network. 


\subsubsection{Defining the Mission}

As briefly discussed in the previous section, processes define the movement of elements and cargo throughout the time expanded network. They define the how and when in the supply chain. The ISCMLA framework defines five core processes [2]. These are:

Exploration processes model exploration and science activity at a specified node. This is typically a surface node, however exploration can also happen at in-space nodes (e.g. in the ISS).

Proximity Operation processes model local rendezvous/docking, undocking/separation, and transposition of elements.

Transfer processes model the transfer of agents and/or supply items. In orbit, transfers must be from one element into a different co-located element. On the surface, transfers can also be between an element and a node, for example a sample return to earth.

Transport processes model the relocation an element to a new physical node along an allowable arc.

Waiting processes model remaining at the same physical node for some number of time periods.

In a given process, the user can dynamically create instantiations of elements from a collection of templates, which are derived from elements used in past and future exploration campaigns such as ISS and Constellation. Alternatively, the user can select from previously instantiated elements and continue using them throughout a scenario. Previously instantiated elements can include the propulsive stages and surface systems delivered to an orbit by a launch vehicle. These elements may be be used in future processes until they reach a surface location. Processes can be grouped into composite processes, which provide a layer of abstraction to simplify scenario building. Typically a composite process encompasses the constituent processes of a sortie, unmanned cargo flight, outpost flight, etc.

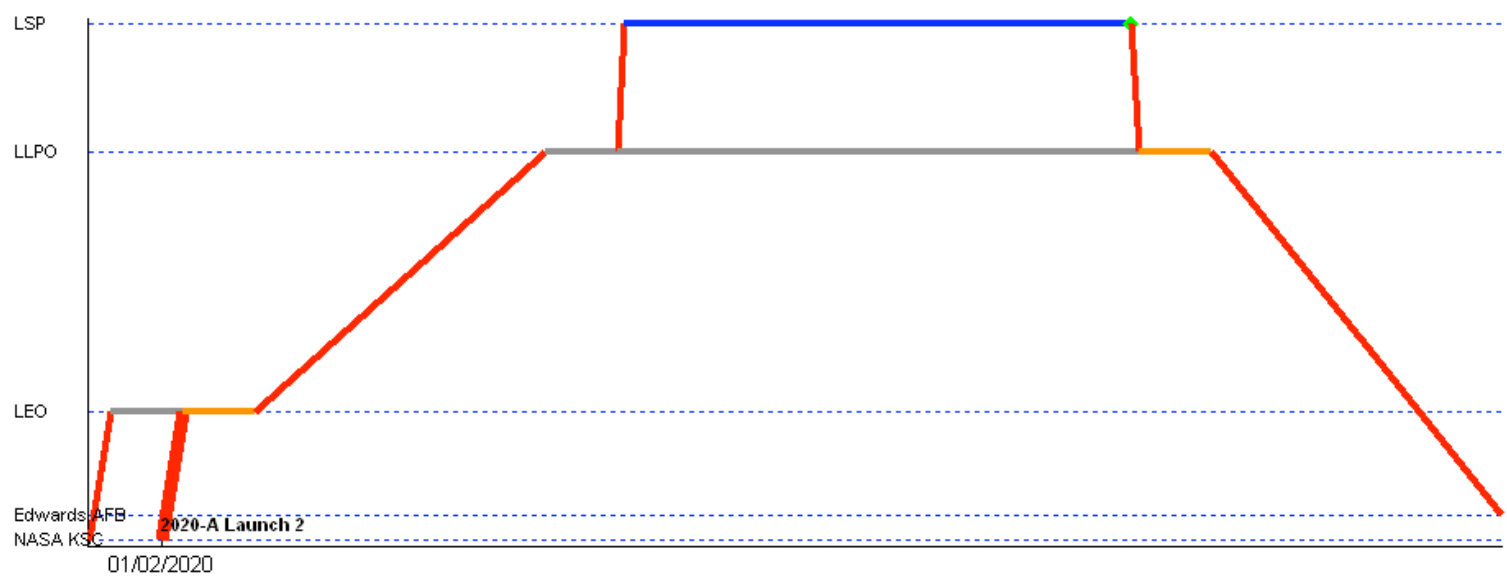

Figure 2-2: Space logistics processes. The horizontal axis represents time, while the vertical axis represents node location. Blue $=$ Exploration, Orange $=$ Proximity Operation, Green $=$ Transfer, Red $=$ Transport, Gray $=$ Waiting. 


\subsubsection{Defining Resource Models}

Generally, demands for habitation and infrastructure $(\mathrm{CoS} 8)$ and transportation and carriers (CoS 9) are defined explicitly as part of the scenario building process. Likewise, demand for fuel and propellants is a result of the definition of demand for CoS 8 and CoS 9 and the process definition. However, demand for the remaining classes of supply is typically complex and dependent on the details of the exploration architecture and must therefore be specified by the user. SpaceNet 2.0 captures these demands through 3 resource models: The demand model, the spares model, and the accommodation model.

Table 2.1: Resource Models and Classes of Supply

\begin{tabular}{|l||c|c|c|c|}
\hline & $\begin{array}{c}\text { Process } \\
\text { Definition }\end{array}$ & $\begin{array}{c}\text { Demand } \\
\text { Model }\end{array}$ & $\begin{array}{c}\text { Spares } \\
\text { Model }\end{array}$ & $\begin{array}{c}\text { Accommodation } \\
\text { Model }\end{array}$ \\
\hline CoS 1: Fuel and Propellants & $\mathrm{X}$ & & & \\
\hline CoS 2: Crew Provisions & & $\mathrm{X}$ & & \\
\hline CoS 3: Crew Operations & & $\mathrm{X}$ & & \\
\hline CoS 4: Maintenance and Upkeep & & & $\mathrm{X}$ & \\
\hline CoS 5: Stowage and Restraint & & & & $\mathrm{X}$ \\
\hline CoS 6: Exploration and Research & $\mathrm{X}$ & & & \\
\hline CoS 7: Waste and Disposal & & $\mathrm{X}$ & & \\
\hline CoS 8: Habitation and Infrastructure & $\mathrm{X}$ & & & \\
\hline CoS 9: Transportation and Carriers & $\mathrm{X}$ & & & \\
\hline CoS 10: Miscellaneous & & $\mathrm{X}$ & & \\
\hline
\end{tabular}

\section{Demand Model}

The SpaceNet 2.0 demand model is a tree-based data structure that allows the user to define arbitrary demand structures for crew provisions ( $\operatorname{CoS} 2)$, crew operations (CoS 3), and waste and disposal $(\mathrm{CoS} 7)$. A demand model is an aggregate of demand variables, of which there are two types: rate variables and reference variables. Rate variables define demand for a particular supply item, a consumption rate, and a unit of measure. Users may select from a variety of parametric probability distributions or use a deterministic value for the rate. Reference variables define demand for a particular supply item in terms of fractions of another supply item. For example, the leakage rate of oxygen and nitrogen in a habitat can be represented as a reference variable that is referenced to the habitat volume.

\section{Spares Model}

The spares model is used to define the demand for spares and related equipment for infrastructure (i.e. elements) specified in the scenario building process. The spares model can be specified in two ways, which are spare-by-mass and spare-to-availability.

The spare-by-mass calculates the mass and volume of pressurized and unpressurized spares for a given time period for an element as a function of its dry mass. Spare-to-availability calculates spares demands as a function of element orbital replacement units (ORUs) and shop replacement units (SRUs). Such demands are application-specific and rely on attributes such as mean-time-betweenfailure (MTBF) and duty cycle. Specifically, sparing by availability calculates a "buylist" of spares needed to meet a desired target availability specified by the user (the default value is $95 \%$ ). The total mass and volume of spares required is then obtained by multiplying the quantity of each ORU in the 
buylist by its mass or volume respectively. SpaceNet returns a buylist that meets the availability target even if it violates the resource constraint (i.e. the amount of cargo mass and/or volume available for delivering spares to the exploration site). In this case, SpaceNet also gives the user the option to recalculate the spares based on the highest estimated availability that is achievable.

While sparing-to-availability is more accurate than sparing-by-mass, it cannot be used until the element ORUs and SRUs are well-defined, which makes it difficult to use in immature exploration architectures where that information is not well known. The spare-by-mass functionality provides a reasonable approximation for spares demands as a temporary measure while an architecture mature and reliable data becomes available.

\section{Accommodation Model}

The accommodation model captures the demand for stowage and restraint (CoS 5) based on the results of the demand model. Users have the option of specifying an arbitrary number of accommodation mappings, each of which pairs a supply item type with a particular packing container type, and sets a packing efficiency for the container type which essentially indicates when to stop manifesting a container and start manifesting a new one. There may be multiple mappings per supply item. For example, food-related supply items might be paired with cargo transfer bags (CTBs) and spares items with SHOSS boxes; water may be paired with a full-size water tank as well as with a smaller crew water container (CWC). SpaceNet 2.0 selects the best containers for each demand based on minimizing the tare fraction and maximizing container capacity utilization. Containers are manifested based on maintaining a clear separation between supply classes and consumers (i.e. elements and agents), for example an individual container may be packed with food and related items for the CEV, but may not be packed with a mix of food and waste disposal equipment for the CEV and LL-D, respectively. This reflects typical real-life manifesting practice.

\subsubsection{Forecasting Demand}

The Forecasting Module (FM) is a basic implementation of the full discrete event simulator that utilizes the demand model to estimate scenario demands based on a target availability. The FM flattens the demand model, parses demand units, and performs the necessary mathematical operations are performed to produce a demand in terms of physical units of weight and volume. The FM has two modes: standard mode and quick estimation mode.

In standard mode the FM receives an event schedule for the simulator corresponding to the desired forecasting horizon. From this point the FM can proceed either deterministically or stochastically. For deterministic estimation, the FM estimates demand by event according to the nominal (mean) value of each demand variable. For stochastic estimation, the FM uses inverse transform sampling, which allows the generation of samples of arbitrary probability distributions [9], to produce an estimate of the demand value for each event. The SpaceNet 2.0 forecasting module currently supports uniform, normal, lognormal, triangular, exponential, Weibull, and Poisson distributions.

Once demands are estimated for each event, either deterministically or stochastically, the FM merges demand by consumer, i.e. the entity that generated the request for resources. If the event schedule spans multiple process groups, demands for each element are further merged by process group. Finally, the forecasting module can optionally pack raw demands into containers based on the accommodation model. Users can also specify a global tare fraction, in which case the FM packs demands into a generic container with the appropriate specifications. 


\subsubsection{Manifesting Demand}

Calculating the demand determines the nominal amount of cargo required to support the surface systems and exploration agents in a campaign. To understand if such a campaign is feasible, and to evaluate what the most effective delivery strategies are, the packed items must be manifested into cargo carriers. Manifesting the discretized supply items into cargo carriers must satisfy multiple constraints. The cargo carrier must have the available pressurized or unpressurized mass and volume capacity for a given supply item. If the cargo carrier, such as a pressurized logistics module (PLM), is nested in another element such as a descent stage, then the cargo capacity of that parent element must be satisfied as well. In addition to the supply items created by the cargo demands from the surface systems and exploration agents, a user can also manifest exploration items such as science instruments, and reserve supplies such as safety stocks. These reserves would serve to hedge against uncertainties in demand or flights that are delayed or cancelled. While the manifesting process can be performed by hand to optimize packing efficiency, it can also be completely automated using a pair of simple heuristics.

\subsection{Simulation}

Simulation ties together all other components of the modeling framework, taking the mission scenario as an input and producing the output information that evaluates both the feasibility and effectiveness of the scenario.

\subsubsection{Discrete Events}

SpaceNet is built on discrete event simulation, whose strength lies in the ability to model random events and to predict the effects of the complex interactions between these events. This flexibility allows various hypotheticals to be investigated by changing inputs to the model and then comparing the outcomes. This makes discrete event simulation a strong decision support tool.

In logistics, the important pieces of information for the user are the states of simulation entities before a given process occurs, the characteristics of the process, and the entity states after the process is completed. For instance, if an element is placed in LEO and then transported to the lunar surface, the key pieces of information are the states of the element and node before the transportation process (mass and crew/cargo of the element, physical node characteristics and time), characteristics of the transportation process (e.g. the departure node, $\Delta V$, time of flight, and arrival node), and information after transportation process (e.g. residual propellant) Thus the space logistics framework is more suited to an event-driven approach than to a time-driven approach.

SpaceNet 2.0, unlike previous versions, redefines the 5 core processes as composites of events, which are the new atomic unit of simulation. The following subsections discuss the modeling and performance advantages of using events as the atomic unit of simulation. At the time of writing the set of events includes:

- Arrival events model the arrival of an exploration resource to a node.

- Departure events model the departure of an exploration resource from a node.

- Explore events model exploration activity such as EVAs and performing of experiments. Typically an Explore event represents a single EVA. 
- Burn events model the firing of main engines and/or reaction control systems in a single element.

- Stage events model the staging (i.e. detachment from an active stack for discard) of an element, usually during a transport process, but occasionally during a wait or proximity operations process.

- Relocate events model the movement of scenario entities from one node to another. This includes surface relocations as well as orbital relocations. Relocation events are composites of arrival and departure events, but include a duration for generating rate-based demands.

- Transfer events model the movement of supply items from one container to another container, where a container is any element or supply item that can hold supply items, and all surface nodes.

Based on the manner in which events are generated, processes can be simulated in more or less detail based on simulator performance or user preference. For instance a user may choose to increase precision for Exploration processes while reducing precision on Transport processes. This enables the user to make better tradeoffs in terms of speed and precision and change his or her preferences in different parts of the same simulation. In addition to enabling variable precision simulation, events also enable custom processes. Exposing some events to the users allows users to design custom processes by combining events as desired. This is particularly important for surface operations which require detailed specification to model accurately.

\subsubsection{Clock Management}

SpaceNet 2.0 introduces a next-event time advance approach, which is the most common approach used in major simulation packages [9]. In contrast, previous versions of SpaceNet used a time step approach. With a next-event time advance approach, the simulation clock is advanced to the next scheduled future event, which is then executed. The system state is then updated and the system clock moves to the new next scheduled future event as shown in Figure 2-3. Concurrent events are made sequential by giving execution preference to the event originating from the earliest initiated process.

The advantage of the next-event time advance approach are reduced simulation time since only time periods with events are analyzed, and also accuracy since events are always executed on time and in sequence (rather than as part of a batch). This improvement in speed and accuracy allows SpaceNet 2.0 to perform simulation runs in the background, providing the user with various pieces of useful data, while supporting implementation of Monte Carlo simulation.

\subsubsection{System State and Histories}

During simulation, each agent, supply item, element, and node is tracked and its history recorded. These histories are used for calculating performance measures, visualization, and user post-processing of simulation data. The 3 types of history are:

EHistory tracks the elements, giving the location and transportation history of each element. It also provides the mass (propellant and dry mass) and crew/cargo information for each element. 
NHistory tracks the nodes and provides information on which elements are located on each node at a specific time.

CHistory tracks crew/cargo and shows which element the crew/cargo belongs to at a specific time.

The three histories have built-in redundancy and form different interpretations of the same mission scenario. Together, the three combine to provide a complete reconstruction of each simulation run. For instance, NHistory, EHistory, and CHistory combine to provide a complete record of the supply levels of each supply class at a given node. SpaceNet 2.0 is capable of outputting each history in Excel and XML formats.

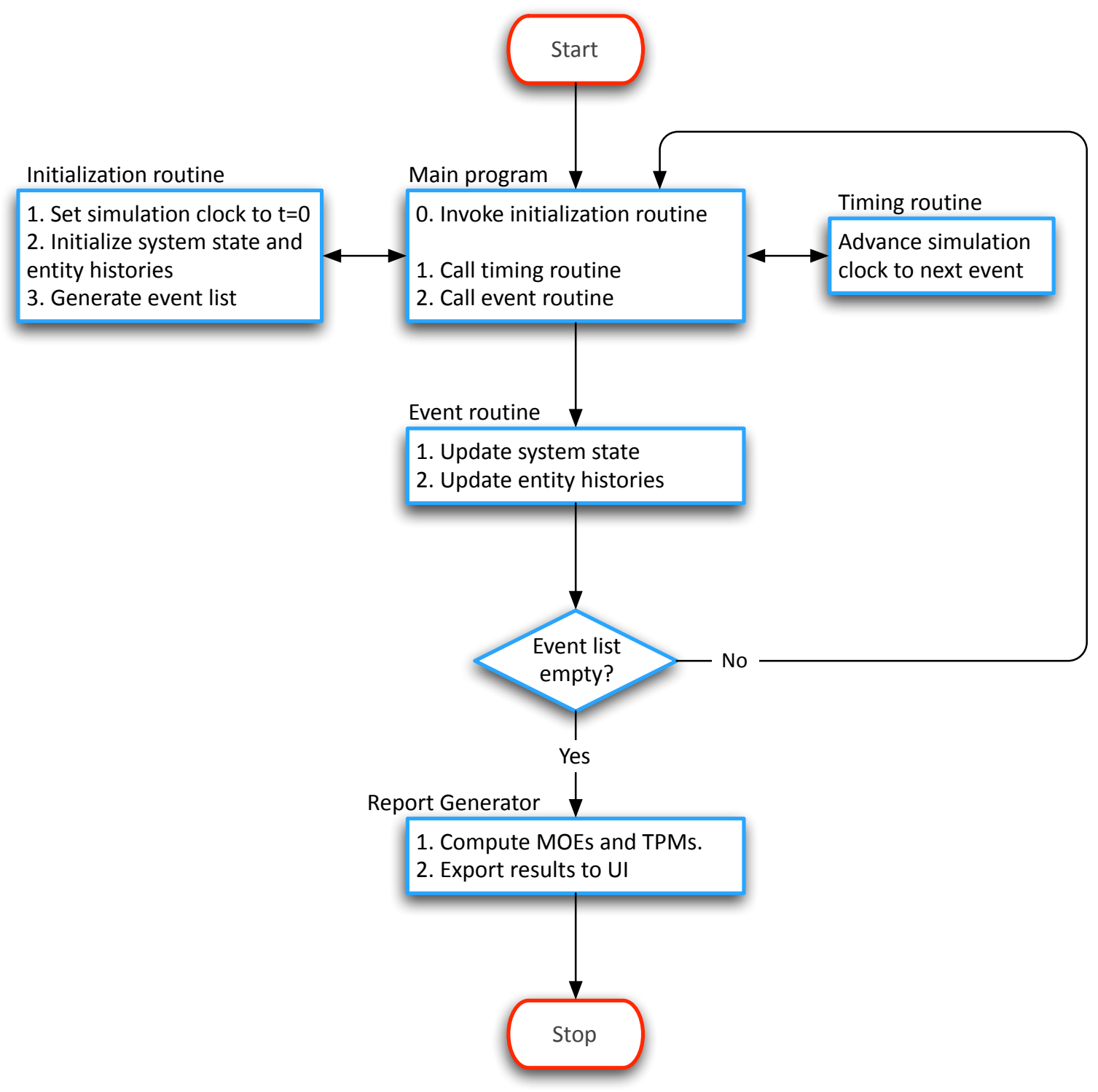

Figure 2-3: SpaceNet 2.0 variable time advance clock management 


\subsubsection{Performance Measures}

An important final step in supply chain design and analysis is the ability to process the simulation output in such a way that architectures can be evaluated and compared [5]. Several metrics (termed measures of effectiveness or MOEs), have been developed hand-in-hand with the simulation and modeling framework to enable the comparative evaluation of logistics architectures [2]. MOEs provide a quantitative way to evaluate specific space exploration scenarios and interplanetary supply chains in general. It is important to note that MOEs are proxy metrics for comparative purposes, not for absolute forecasting. MOEs continue to evolve to better capture new exploration paradigms and the benefits derived from unmanned activities such as the presence of robots on planetary surfaces and orbiting spacecraft observation. MOEs fall into three broad categories:

Basic Logistics Performance: Any mission's performance is largely defined by the capability of the launch vehicles and space craft involved, as well as their utilization. The basic logistics performance MOEs are designed to quantitatively measure capability and utilization, thereby providing the user an performance envelope within which to modify and optimize the distribution of mass between supply classes as desired.

Exploration Capability: The true benefit (revenue) of planetary space exploration is not monetary, but comes primarily from healthy and qualified explorers, scientists, and robotic systems (i.e. agents) being able to spend a certain amount of time at one or more nodes. To first order, revenue scales linearly with both the number of agents as well as the duration of their stay. In addition, specific exploration equipment, scientific instruments, and surface infrastructure items play a large part in facilitating exploration, and act as multipliers for exploration productivity. The exploration capability MOEs are designed to capture this concept.

Relative Cost and Risk: To first order the cost of a scenario scales with duration (operating and observation costs) and launched mass (development and recurring costs). The actual details of both risk and cost however are extremely complex and beyond the scope of the logistics modeling framework. Thus the the Risk and Cost MoEs relate risk and cost in these relative terms without attempting to define a precise probability distribution for risk and/or an absolute monetary value for cost.

Appendix A provides detailed equations of the measures of effectiveness.

\subsection{Data Management}

In an attempt to improve asset management for human space-flight missions to the Moon and Mars, a relational database for interplanetary exploration, aptly named the Interplanetary Supply Chain Management (ISCM) database has been developed. This database incorporates inventory management capabilities with extensive capabilities for manifesting, spares requirements planning and mission planning. Information maintained in the integrated database includes astrodynamics data, element data, commodities data, and spares data. SpaceNet uses an enhanced version of the ISCM database to store all the information required by the model, as well as to store in-process data as the user designs a scenario. 


\subsection{Chapter Summary}

This chapter has described an interplanetary logistics modeling and simulation tool, SpaceNet 2.0, developed by systems engineers at JPL and MIT. The goal of SpaceNet is to allow mission architects, planners, systems engineers and logisticians to focus on what will be needed to support future crewed exploration missions, primarily in the Earth-Moon-Mars system. Instead of helping to design the elements (vehicles) themselves in terms of propulsive and pressurized/unpressurized cargo carrying capability, SpaceNet evaluates such vehicles in the context of a particular mission architecture and supply chain strategy. The emphasis is on ensuring the logistical feasibility of a given scenario as well as a prediction of the resulting logistics measures of effectiveness (MOEs). All this is enabled by detailed simulation of time-varying flow of elements, crew and supply items through the nodes and arcs (trajectories) of a supply network in space, taking into account feasibility ( $\Delta V \mathrm{~s}$, fuel levels) as well as consumption and supply. 


\section{Chapter 3}

\section{Revenue Management}

\section{$3.1 \quad$ Overview}

Revenue Management (RM) is the optimization of the revenue or benefit earned from a fixed, perishable resource [10]. Extending the concept are flexible products, which are defined as a menu of two or more alternatives, typically substitute, products (or services) offered by a constrained supplier using a sales or booking process [11]. Airlines, hotels, and rental cars represent the traditional "big three" applications of revenue management. Although researchers have applied revenue management models in a wide variety of industries where suppliers offer flexible products, these three industries still dominate the conceptual framework of revenue management. The following sections provide overviews of the RM problem in each of these industries, including the unique features of each. Section 3.1.5 also provides a brief overview of the extension of revenue management into new areas. What one can see from these examples is how flexible RM can be in practice.

\subsubsection{History}

Research on revenue management can be traced back over 40 years to 1966, when American Airlines implemented the SABRE computer reservations system. However, only after the Airline Deregulation Act of 1978, which relaxed pricing controls, did RM become prevalent in the airline industry [12]. Since then significant innovations have been made. Initially, airline revenue management systems were only capable of handling single-leg, origin-destination flights. New information technologies have led to more sophisticated revenue management capabilities, while at the same time theoretical advancements have allowed RM to spread beyond the airline industry. Today revenue management systems and related information technologies are heavily used by airlines, hotels, car rentals, ecommerce, and many other industries. In low margin, high fixed cost businesses like airlines and hotels, this increased revenue almost directly translates into increased profits, while maintaining the quality of service to the customer [13].

\subsubsection{Airlines}

For the airline industry, the RM challenge is essentially identical to the RM challenge stated in Section 1.3, that is to sell the right resource(s) to the right customer at the right time in order to maximize revenue. The nature of the airline industry means that this translates into a series of 
booking decisions - whether or not to accept a current booking request or wait for possible future higher revenue requests. Typically the airline is modeled as a risk-neutral entity, which has been shown to be an accurate model given that booking decisions are made thousands of times per flight and millions of times per year [10].

As of 1998, it is estimated that revenue management was directly contributing USD 500 million and 300 million in increased annual revenues and earnings to US Airlines and Delta Airlines, respectively [14]. Since, the market has evolved to be even more conducive to revenue management. Given that airlines have consistently posted losses over the last decade, one may ask if revenue management is truly effective. However, it is important to note that airlines suffer these losses due to the inherent nature of the highly competitive markets they operate in. Within those constraints, revenue management helps to increase revenues and profits (i.e. minimize losses).

\subsubsection{Hotels \& Rental Cars}

Revenue management of hotels has some differences from the airline case. One of the differences is that hotels face uncertainty in terms of how much of the resource each customer will consume [15]. In other words, customers can make multiple day reservations and are relatively free to change the duration of their stay on short notice. Although multiple day reservations can pose problems, Ladany suggests that for many hotels they represent only a small portion of total reservations. Rothstein modeled the multi-day problem as a series of independent, single night reservations by assuming that the individual dates of a multi-day reservation cancel or show-up independently [16]. Bitran, Gilbert and Leong present empirical evidence which validates this assumption [17]. It is estimated that revenue management helps Marriott Hotels to gain USD 100 million in additional revenue each year [18].

The rental car industry, like the hotel industry, also faces a network consumption problem. However, another unique aspect aspect of the rental car industry is the ability to increase capacity at a relatively reasonable cost as well as some ability to reorganize the fleet to meet demands. Rental car companies also feature a much more homogeneous customer population - most renters are satisfied with a relatively basic mobility option (a fact reflected by fleet compositions) [19].

\subsubsection{Cargo}

The air cargo industry shares many common characteristics with airlines, although in general its revenue management framework is significantly less developed than that of the airline industry. Still, cargo revenue management differs from the airline revenue management problem in several ways. First, the resource is multi-dimensional; in contrast to a seat on a passenger airline, cargo is defined in terms of both weight and volume. Secondly, each customer is unique and requires a different amount of the resource; in other words every customer's shipment is a unique weight and volume. Thirdly, the amount of the resource for sale is often unknown due to the nature of the cargo industry. For most carriers, allotments (space reserved for big customers) take up much of the cargo space. The sum of unused allotment space and unreserved space is a random variable and forms the resource available for spot sale (i.e. application of revenue management). Finally, cargo shippers typically have an ample supply of standby cargo available, which essentially guarantees that no resource unit is left unused. In this case, standby cargo can be modeled as the lowest class of cargo with infinite demand $[20]$. 
Cargo, and in particular air cargo, is one of the main growth areas in revenue management, driven by increasing demand for (and revenue from) cargo shipments, in large part a result of globalization.

\subsubsection{Other Industries}

Because of revenue managements success in the "big three" industries, and its growth in the cargo industry, RM has spread to other industries such as restaurants, casinos, internet services and apartment rentals [12]. Coulter suggests that revenue management is appropriate in any seasonal retailing industry in which capacity (inventory) is not necessarily perishable but the value of the capacity may decline significantly after the selling season [21]. He goes on to investigate the use of discount pricing to maximize the revenue gained from selling a seasonal product. Investigating seasonal products further, Aviv and Pazgal perform a quantitative analysis on how to apply dynamic pricing to sell fashion-like goods for seasonal retailers [22]. Hatwin, Lippman focus on issues such as pricing strategy, market share preservation, and customer loyalty when applying revenue management techniques on grocery retail outlets [23][24].

Revenue management also has application opportunities in subscription services, such as ondemand information technology service and Internet service. Internet service is, in fact, a special case of on-demand information technology service. Nair and Bapna find that Internet Service Providers (ISP) have perishable capacity for users to $\log$ on, a fixed number of units, and the possibility of segmenting price-sensitive customers [25]. One unique feature of the ISP case is that the service is continuous in state and time, i.e. the request and the service happen simultaneously.

\subsection{Revenue Management Disciplines}

Within the field of revenue management, there are four main areas of development: forecasting and estimation, overbooking, pricing, and capacity control.

Forecasting and Estimation is concerned with gathering of information and the formation of accurate models of demand for each flexible product offering.

Overbooking is concerned with increasing utilization of the resource as much as possible while maintaining the level of service.

Pricing is concerned with the setting and manipulation of prices.

Capacity control concerns the allocation of the resource between the various products (resource bundles for sale).

The following sections give a conceptual overview of and a brief summary of key research in each of the four areas, with a special focus on capacity control, which is the most applicable discipline to space logistics. In addition each section discusses the potential of the application of various solution approaches to space logistics RM. These findings constitute the conceptual foundations of the SpaceNet RM module presented in Chapter 4.

\subsubsection{Forecasting and Estimation}

Every revenue management system requires some sort of forecast or estimate of demand. More specifically, forecasting predicts demand from existing data, while estimation seeks to characterize 


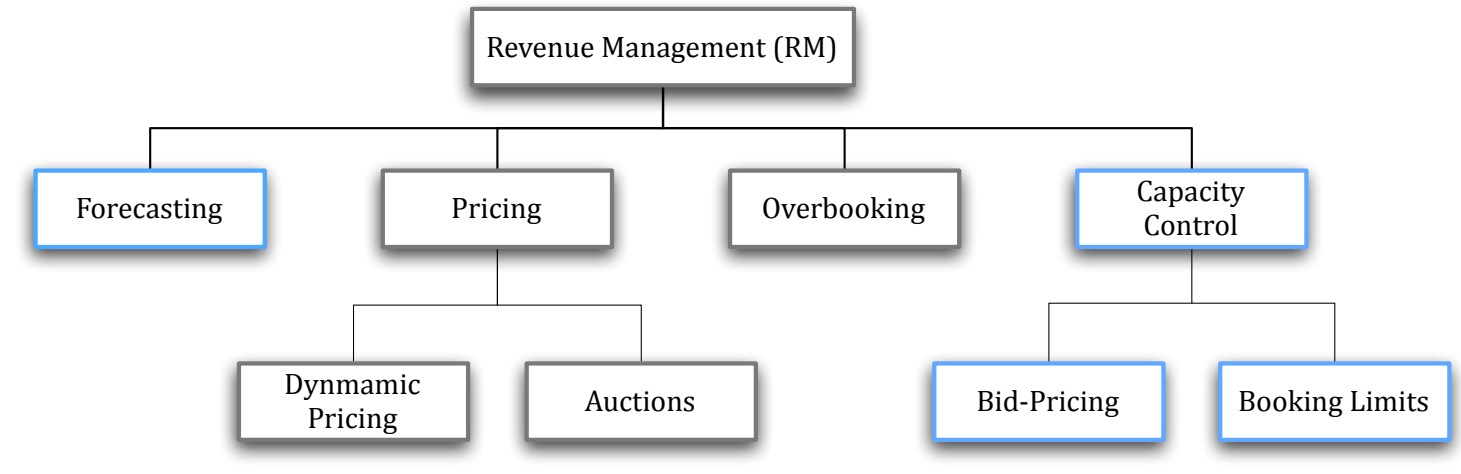

Figure 3-1: Overview of revenue management practice

observed data and form a model. Typically, revenue management systems use both estimation and forecasting; estimation is used to calibrate forecast parameters. Depending on the use of pricing, capacity control, and/or overbooking, forecast requirements can expand to price sensitivity, segment demand, and/or cancellation probabilities, respectively. Any model is only as good as its data, and revenue management is no different. Some industry estimates suggest that a 20 percent reduction in forecast error can lead to an incremental 1\% increase in revenue [10]. Given that industries that use RM are typified by high capital costs and low profit margins, this relatively small increase can have a significant impact on the bottom line.

Consider a basic airline revenue management problem, where the airline has decided on a two class fare structure with prices at 100 and 70, respectively (assume the prices are determined using some offline pricing model). Table 3.1 shows an example of the type of output a basic forecast might produce. This includes an estimate of mean demand, and a standard deviation based on an assumed distribution. A more advanced forecast might also include a temporal element that estimates he arrival profile of each class.

Table 3.1: Basic demand forecast with prices, two-class example

\begin{tabular}{|c||c|c|c|c|}
\hline & Price & Mean & Std. Dev. & Distribution \\
\hline Class 1 & 100 & 10 & 5 & Normal \\
Class 2 & 70 & 20 & 5 & Normal \\
\hline
\end{tabular}

\subsubsection{Pricing}

In price-based revenue management, the producer uses prices to manage demand. For many industries, varying prices is the most natural mechanism for implementing revenue management, and indeed dynamic pricing can be considered the oldest form of revenue management, pre dating the formal practice itself [10]. The key difference in price-based revenue management compared to quantity-based revenue management (i.e. capacity control) is that demand is as an endogenous variable.

Pricing controls come in two categories - dynamic pricing and auctions. Dynamic pricing includes markdowns, sales, coupons, discounts, and personal pricing. Auctions include the various types of auctions (e.g. open English and open Dutch) as well as requests for proposals. Within the two 
categories, there are a host of deterministic and stochastic solution techniques. Deterministic solutions to the pricing problem can be obtained by using standard optimization techniques, nonlinear programs, or greedy algorithms. The stochastic solution of a pricing problem can be acquired by using a variety of approaches including action-space-reduction, Markov decision processes, and the Hamilton-Jacobi-Bellman (HJB) equation [26].

The applicability of price-based revenue management in space logistics appears to be relatively limited. In space logistics demand is almost always an exogenous variable. That is the demand for the resource is independent of the producers pricing control; rather it is driven by the demand models of the agent. The question is to determine how best to allocate the resources to those demands, which is closely aligned with the quantity-RM problem.

\subsubsection{Overbooking}

Overbooking is the oldest tactic in revenue management [12]. While pricing and capacity allocation are essentially concerned with how to achieve the best mix of demand (from a revenue perspective) overbooking is concerned with increasing capacity utilization in RM problems with a high possibility of cancellations or no-shows. American Airlines estimates that $15 \%$ of seats on sold-out ights would be lost if overbooking were not practiced [27]. In 1990, the benet of overbooking at American Airlines was estimated to exceed USD 225 million [27].

Overbooking models typically take either a cost based approach (i.e. they balance the cost of overselling with the opportunity cost of empty seats) or a service level based approach (i.e. they balance the expected number of passengers denied service with the cost of empty seats) [12]. Beckmann provides a static, single period cost-based model, to determine an upper bound on the number of reservations to accept [28]. Thompson shows a way to determine overselling probabilities for a static, single-leg problem [29]. In addition, Chatwin, Rothstein, and Subramanian et al. have addressed dynamic models of overbooking for single-leg ights [30] [31] [32].

While overbooking is a critical part of revenue management for airlines, hotels, and rental cars, it is not the most applicable RM discipline for space logistics. In space logistics, the danger for the producer is not empty seats due to no-shows and cancellations, it is a loss of revenue due to "no-pays", i.e. a supply item that is unable to contribute to the exploration return of the mission.

\subsection{Capacity Control}

As previously discussed, ultimately the goal of space logistics is to find the best allocation of mass and volume for the various classes of supply to maximize exploration benefits, minimize risk, etc. Thus capacity control, and in particular single-resource capacity control (to be discussed later) is perhaps the most applicable discipline to space logistics. There are several concepts that arise throughout virtually all of capacity control:

Products are restricted resource bundles that segment the market. The product is constrained by a maximum quantity, $C$, that is available for sale.

Booking limits, $b$ set the maximum amount of the resource units that can be sold to a particular class at a given point in time.

Protection levels, $y$ are the complementary concept to booking limits. They set a minimum amount of the resource to protect for higher fare demand. The relationship between booking 
limits, $b$, and protection levels, $y$, is given by:

$$
b_{j}=C-y_{j-1}
$$

where the booking limit for a given class includes the resources available in higher classes. Consider in the demand scenario shown earlier in Table 3.1, given a capacity of 25 . In this case

$$
b_{2}=25-y_{1}
$$

and if Class 1's protection level is 15, Class 2's booking limit would be 10 .

Partitioning places the resource allotment (defined either in terms of booking limits or protection levels) for each class in separate, exclusive buckets.

Nesting overlaps booking limits or protection levels in a hierarchical manner, thereby ensuring each fare class has access to all of the buckets from lower fare classes. Because of the hierarchy, nesting avoids one of the major pitfalls of partitioning, which is simultaneous unavailability of resource units for high fare classes and availability of resource units for low fare classes. This makes it more favored in practice [10].

\subsubsection{Levels of Decision Making}

There are three levels of decision making in capacity control: strategic, tactical, and booking [33]. Strategic control concerns market segmentation and price differentiation; essentially the goal is to precisely identify customer segments, or classes, and developing mechanisms to segment the market. Tactical control concerns dividing resource units among the different market segments. Finally, booking control concerns the real time decision whether or not to acceptance booking requests. In this thesis we focus on tactical control. While the implementation of optimal capacity controls can be complex, including a mix of strategic, tactical, and booking elements, the overriding logic is simple - capacity should be allocated to a request if and only if its revenue is greater than the value of the capacity required to satisfy it. Secondly, the value of capacity should be measured by its expected opportunity cost, as determined by a value function $V(x)$, which measures the optimal expected revenue as a function of the remaining capacity $x[10]$.

Single-resource capacity control problems were first solved by Littlewoods rule [34]. Following that breakthrough, the decision tree approach, expected marginal seat revenue (EMSR) heuristics (EMSR-a, EMSR-b, and modified EMSP heuristics), bid pricing methods, adaptive methods [35], and Markov-based reinforcement learning [36] have been proposed as solution techniques. Virtual nesting booking control, network bid pricing method, and dynamic virtual nesting have all been used to model the network capacity control problems. These problems have been solved by linearprogramming approaches, approximate dynamic programming [37], and scenario tree approaches [38]. Other approaches use polyhedral graph theory, reinforcement learning, machine learning, hierarchical mathematical optimization, Robust optimization, decision trees, and stochastic knapsack $[12]$.

The following sections explore a few key controls and solution methods for the single resource problem. While some of these examples are relatively basic and not necessarily at the cutting-edge of research, they clearly present the key concepts of capacity control. Thus they form a good basis for a new application (space logistics). 


\subsubsection{Littlewood's Rule}

One of the earliest models for single-resource capacity control was developed by Littlewood [34] and is similar to the newsboy problem in inventory management. The model featured two product classes with prices $p_{1}>p_{2}$, production capacity $C$, and the following assumptions:

1. Low revenue customers ask for resource units before high revenue customers

2. Demand from one class is independent from the other

3. The probability distributions of arrivals is continuous

4. There are no cancelations of realized bookings

Littlewood determined that class 2 requests for resource units should be accepted as long as

$$
p_{2} \geq p_{1} \cdot P_{j}\left(D_{1}>x\right)
$$

where $D_{j}$ is the demand of class $j$ with distribution $P_{j}(\cdot)$.

Equation 3.3 is known as Littlewood's Rule, and it suggests that the optimal protection level for class 1 should be set so that

$$
p_{2}=p_{1} \cdot P\left(D_{1}>y_{1}^{*}\right)=E M S R_{1}
$$

where $E M S R_{1}$ is the expected marginal seat revenue from the high-fare class.

In the case of the demand forecast in Table 3.1, Littlewoods rule would find that the optimal protection level of class 1 from class 2 should be set so that

$$
\begin{aligned}
P\left(D_{1}>y_{1}^{*}\right) & =\frac{70}{100} \\
y_{1}^{*} & =22.6
\end{aligned}
$$

Littlewood's model, and in particular the EMSR criterion, show that at the heart of capacity control is the decision to accept or reject a certain request low fare customer or wait for an uncertain request from a higher fare customer. Bhatia and Parekh [39] later proved that Littlewood's criterion is in fact the revenue maximizing condition for the two class problem. Richter [40] also verified Littlewood's rule through the concept of "differential revenue" $R_{D}$ which was defined as:

$$
R_{D}=p_{2}-E M S R_{1}
$$

One can see from Equation 3.3 that the capacity allocation only depends on the probability distribution of the high-fare demand (and of course the ratio of the fares). This is the result of the assumption that low-fare demand customers book first, and therefore their demand is known with certainty at the time of decision. However, it has been shown that through simulation that Littlewood's rule performs well even if the arrival order is only approximately low to high demand [41]. More specifically, Mayer extended the model to a series of booking periods where in each period low fare customers book before high fare customers [42]. He found that use of Littlewood's rule lead to only a slight decrease in revenue compared to more complex optimization methods. 


\subsubsection{Expected Marginal Seat Revenue}

While the two class model provides significant insight into the core concept of capacity control, it is limited and somewhat unrealistic because markets can often be segmented into many more than two classes. Belobaba showed that the multi-class problem can be treated as a series of two-class problems, each using Littlewood's rule [43][44]. Then we consider two related approaches to nesting, which are referred to as EMSR-a and EMSR-b.

EMSRa (expected marginal seat revenue-version a) works by aggregating protection levels for a given class $k$ over all higher fare classes 1..j as shown in Equation 3.5.

$$
y_{j}=\sum_{k=1}^{j} y_{k}^{j+1}
$$

EMSR-a will set protection levels so that

$$
P\left(D_{k}>y_{k}^{j+1}\right)=\frac{p_{j+1}}{p_{k}}
$$

for each class $k=1 . . j$. Because EMSR-a adds the individual protection levels $y_{k}^{j+1}$, it ignores the statistical averaging effect produced by aggregating demand across classes. As a result, EMSR-a is sometimes excessively conservative and produces protection levels that are larger than optimal in certain cases [10].

In contrast, EMSR-b (expected marginal seat revenue-version b)works by creating an artificial class that is a demand-weighted average of all the classes 1..j-1 as shown in Equation 3.6. From there, the problem simplifies to the standard two-class model with the artificial class as the high fare class.

$$
p_{j}=\frac{\sum_{k=1}^{j-1} p_{k} E\left[D_{k}\right]}{\sum_{k=1}^{j-1} E\left[D_{k}\right]}
$$

To apply EMSR-a and EMSR-b, we must extend the model and demand forecast in Table 3.1 to include a third class with a price and demand distribution as shown in Table 3.2.

Table 3.2: Basic demand forecast with prices, three-class example

\begin{tabular}{|c||c|c|c|c|}
\hline & Price & Mean & Std. Dev. & Distribution \\
\hline Class 1 & 100 & 10 & 5 & Normal \\
Class 2 & 70 & 20 & 5 & Normal \\
Class 3 & 42 & 30 & 5 & Normal \\
\hline
\end{tabular}

In practice the performance of the two nesting approaches depends on the specifics of the problem structure. One study showed that EMSR-b is consistently within $0.5 \%$ of optimal, while EMSR-a could be as much as $1.5 \%$ below optimal [45]. However, other studies have shown that the approaches perform similarly and neither is universally superior [45].

\subsubsection{Dynamic Controls}

Littlewood's Rule and EMSR are examples of controls with static optimality criterion. In contrast, requests for capacity are dynamic in nature. One can make static models pseudo-dynamic by frequently updating demand forecasts and re-optimizing the booking limits and/or protection levels. However there are a class of dynamic models which explicitly account for the dynamic na- 
ture of capacity allocation. These allow for arbitrary arrival patterns including interspersed class arrivals. However, they are often intractable, unless Markovian demand is assumed [10]. Therefore dynamic models essentially trade one major assumption (arrival sequence) for another (inter-arrival probability distribution).

Brumelle and McGill present an approach based on sub-differential optimization within a stochastic dynamic programming framework to determine optimal booking policies for multiple fare classes for a single flight leg [46]. In a series of articles, Kleywegt and Papastavrou investigate what they call the dynamic stochastic knapsack problem [47][48][49]. They mention its application to cargo revenue management. However, their models include only one capacity restriction. They choose a dynamic programming approach to the problem which, although theoretically very interesting, is computationally very demanding.

\subsubsection{Bid Pricing}

Bid pricing is a revenue management method where threshold values (bid prices) are set for capacity on each flight (or hotel room or rental car), and a seat (or room rental or car rental) is sold only if the offered price is higher than the bid price. However, the bid price is influenced by factors such as the remaining capacity and time to production, and thus bid-pricing is actually somewhat of a hybrid between pricing and capacity control. Bid-pricing is appealing on intuitive and practical grounds - it requires the storage of only one variable (the bid-price) in the control system and is conceptually simple. At the same time, the theory underlying bid-pricing is not well developed nor is the extent to which bid-prices are optimal well understood. Talluri and van Ryzin discuss several different methods for producing bid prices [50].

Bid prices are particularly attractive in network revenue management problems because of their relative simplicity. For example, in the airline case, bid-prices for each leg of a flight can be aggregated to form a bid-price for a given O-D fare. Other controls typically rely on nested fare classes which are capacity allocation problems within a capacity allocation problem and can therefore be quite complex.

\subsection{Implementation}

Figure 3.4 shows the elements of RM system for a legacy carrier in the airline industry. It uses capacity allocation and overbooking, but not dynamic pricing. An RM system, however, can be much simpler than the one shown. To implement a basic revenue management system, one needs to do four things:

1. Collect and store relevant historical data including prices, demand forecasts, and causal factors

2. Find the optimal set of controls (allocations, prices, overbooking) to apply to the next reoptimization

3. Periodically update the optimized control

4. Control the sale of resources using the optimized control (manifesting module)

A large part of the degree to which revenue management is successful in various industries is the ability to implement the theoretical framework into business operations. For example, forecasting 
demand based on historical data is difficult in the restaurant industry, but relatively easy in the airline industry due to the large amounts of government-required documentation.

The next chapter presents an implementation of revenue management for space logistics that utilizes the ISCM database for historical data, SpaceNet 2.0 for demand forecasting and estimation, and a new purpose-built module for optimization and control.

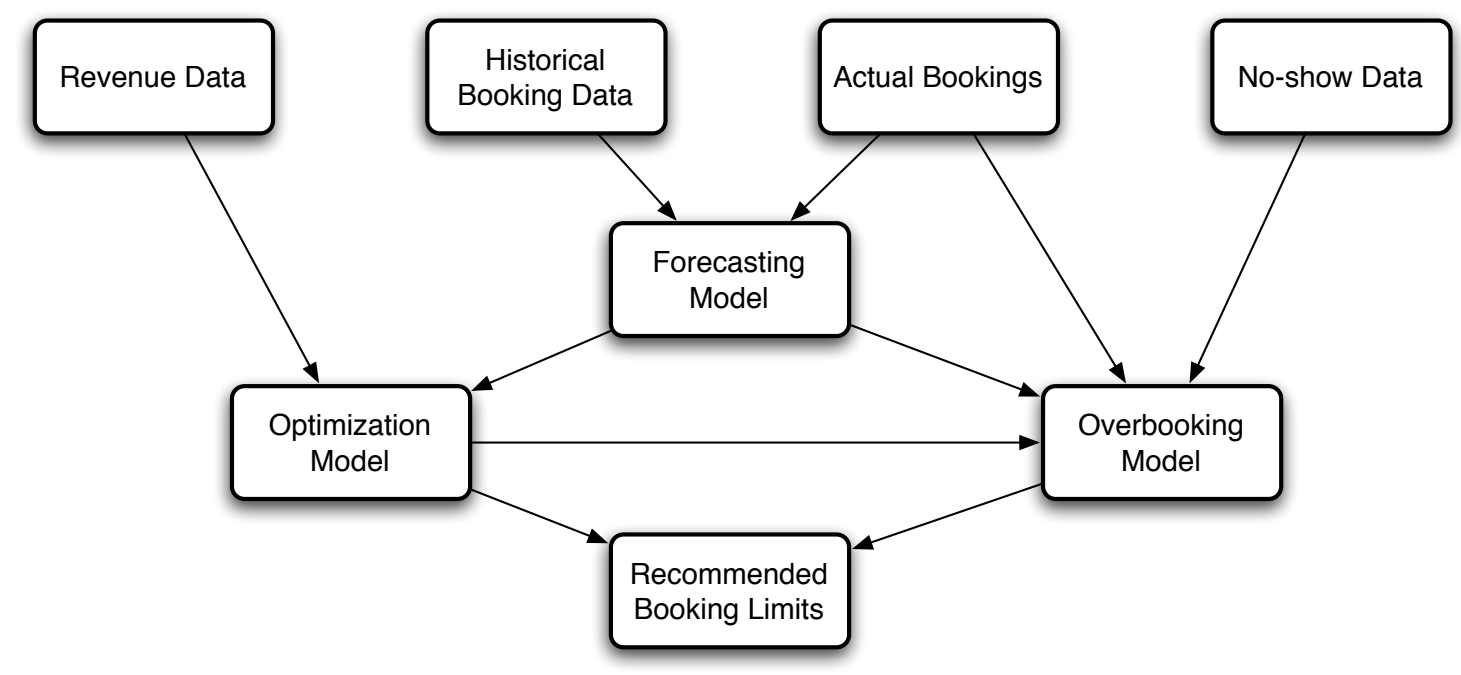

Figure 3-2: Typical airline revenue management system

\subsection{Chapter Summary}

This chapter provided a brief overview of the history of and current practices in revenue management. Of the four primary disciplines in revenue management, capacity control is most suited to space logistics because it deals primarily with allocation of a fixed resource to maximize some objective function. Within capacity control, there are several solution approaches ranging form relatively simple heuristics to complex non-linear optimization schemes. This chapter focused on heuristic approaches, namely Littlewood's rule and the Expected Marginal Seat Revenue (EMSR), and bidpricing which is a hybrid of capacity control and dynamic pricing. These heuristics show that at the heart of every revenue management problem is a probabilistic assessment of the value of each unit of capacity, and whether or not a customer's offer for that unit of capacity exceeds the opportunity cost of accepting that request.

To implement revenue management, one needs to be able to predict, collect, and store demand data, implement a control that governs the acceptance (or rejection) of customer requests, and periodically update that control. As discussed in detail in Chapter 2, SpaceNet 2.0 already features extensive forecasting capabilities through the various resource models and the forecasting module. Additionally, it features extensive data collection and management through the integrated database and detailed entity histories generated through simulation. Thus it provides a strong foundation upon which to implement a revenue management system. The following chapter details the implementation of revenue management in SpaceNet. 


\section{Chapter 4}

\section{SpaceNet Revenue Management}

\subsection{Conceptual Framework}

The Exploration Capability (EC) MOE provides a good starting point for the definition of exploration revenue. At a given node, it is essentially the dot product of crew and exploration support equipment over time:

$$
E C=\Delta T \sum_{i=1}^{T}\left(1-\alpha_{i}\right) N_{c r e w, i}\left(m_{C O S 6, i}+m_{C O S 8, i}\right)
$$

However, as the name suggests, exploration capability captures the capability of a given scenario, not its true performance in a stochastic environment. In other words, it captures the potential of the exploration architecture - exploration support equipment, habitation and infrastructure, and crew - while assuming a certain level of availability for the remaining classes of supply. If not enough of any of these supply items is manifested to achieve this availability, the exploration capability metric remains unchanged. Instead, a resource error is generated and directed to the analyst for resolution (usually resolution involves small modifications to the mission profile).

\subsubsection{Exploration Value}

Exploration Value (EV), as shown in Equation 4.2, is a metric derived from EC that translates resource errors directly into reduced mission performance through a reduction in the availability of crew, exploration payloads, and/or infrastructure. Blanchard gives a qualitative definition of availability as "a measure of the degree to which a system is in the operable and committable state at the start of mission when the mission is called for at an unknown random point in time" [51]. And NASA defines system availability as the ability of the system to operate whenever called upon to do so [52]. Thus system availability can be thought of as the opposite of downtime and is typically expressed as a number (or percentage) between 0 and 1 (0\%-100\%). Exploration Value, is therefore defined as the dot product of exploration capability and the availability of agents and infrastructure to exploit that capability over time:

$$
E V=\Delta T \sum_{i=1}^{T} N_{\text {crew }, i}\left(m_{C O S 6, i}+m_{C O S 8, i}\right) \cdot A_{C R E W, i} \cdot A_{I N F, i}
$$


where $A_{s, i}$ is the availability of a given component of the exploration system $s$ at time $i$.

\section{Crew Availability}

Crew availability is explicitly a function of the number of crew at the exploration site and the fraction of time each crew member can devote to exploration activities, $\alpha_{i}$ [53]. It is also therefore implicitly a function of availability of the various supply items (including crew provisions, crew operations equipment, waste management) which are needed to maintain crew health and safety. Crew availability, $A_{C R E W, i}$, at an exploration site at a given time can informally be defined as:

$$
A_{C R E W, i}=\cdot f\left(\alpha_{i}, m_{C O S 2, i}, m_{C O S 3, i}, m_{C O S 5, i}\right)
$$

The amount of time the crew can devote to exploration activity, $\alpha_{i}$, depends on the detailed exploration architecture including the nature of micro-logistics and surface operations, and exercise, health, and environmental concerns. Intuitively, one can also imagine that a shortage in crew provisions will necessitate that the crew operate at reduced capacity and/or reduce the duration of their mission thereby reducing their availability for exploration activities over the scheduled length

of the mission. To capture this concept we define $V_{i}$ as the event that there are enough provisions to maintain the crew at time step $i$. Crew availability can now formally be defined as:

$$
A_{C R E W, i}=\cdot \alpha_{i} \cdot P\left(V_{i}\right)
$$

\section{Infrastructure Availability}

Infrastructure availability is primarily driven by the spares requirements. A shortage in spare parts for infrastructure reduces mission performance either because it requires the crew to cancel planned exploration activity to perform repairs (rather than replacements), or in an extreme case to abandon the mission. Bachman and Kline [54] developed a hybrid parametric-analytical model for estimating the required spare parts requirements for exploration of the Moon, Mars, and beyond. In this model, as showin in Figure 4-1, demand (failures) occur according to a Poisson distribution with arrival rate. Based on this failure distribution, the number of broken units not yet replaced, or the pipeline, with mean $\lambda$ can be computed. This then translates to a backorder $E B O$ of spares, which can be used to determine availability based on the equations below.

$$
\begin{aligned}
\lambda_{i} & ={\text { demands } / \text { day }_{i} \cdot q_{i} \cdot \text { duration }_{e}}_{\text {der }_{i}(n)}=\frac{\exp ^{-\lambda_{i}}-\lambda_{i}^{n}}{n !} \\
E B O_{i}(s) & =\sum_{n>s}(n-s) p_{i}(n)
\end{aligned}
$$

At any given time, any unit with no failed components is considered available. If failures of item $i$ in different installed locations are assumed to be independent, the probability that no randomly selected element of type $e$ is missing an item of type $i$, and is therefore unavailable is

$$
A_{I N F, i}\left(s_{i}\right)=\left(1-\frac{E B O_{i}(s)}{q_{i}}\right)^{q_{i}}
$$


Finally, Bachman and Kline's model treats failures of different types of items as independent, and thus the probability that a randomly selected element of type $e$ with multiple spare types is

$$
A_{I N F, i}\left(s, s_{1}, \ldots, s_{m}\right)=\prod_{i=1}^{m}\left(1-\frac{E B O_{i}(s)}{q_{i}}\right)^{q_{i}}
$$

The above model estimates availability for complex systems with an arbitrary number of spare types and elements. On average, an exploration system will have tens if not hundreds of different types of spares, each of which has a characteristic MTBF, mass, volume, and criticality. Typically, this large number of spares are grouped into orbital replacement units (ORUs) and/or shop replaceable units (SRU) in the element. For the purposes of revenue management, the ORUs and SRUs are further abstracted into two key types which are 1) pressurized units and 2) unpressurized units. Within these types, an artificial "typical ORU" with mass, volume, and failure rate characteristics is defined using a demand weighted average of the specifications of the various ORUs and SRUs needed to complete a repair.

Figure 4-2 availability-versus-resources curve for a typical ORU. Every point on the curve represents the minimum spares resources required to achieve the corresponding system availability. From the curve it is evident that the marginal contribution to availability provided by an incremental spare changes as the length of the pipeline changes. This in turn suggests that the marginal contribution to exploration value changes in a similar manner. And this variation is what revenue management can exploit to improve logistical performance.

\subsubsection{Marginal Exploration Value}

Given the EV formulation, Marginal Exploration Value (MEV) metric can also be defined for a given class of supply as follows:

$$
M E V_{k}=\frac{\partial E V_{t o t}}{\partial m_{C O S_{k}}} \approx \frac{E V_{t o t}^{b}-E V_{t o t}^{a}}{m_{C O S_{k}}^{b}-m_{C O S_{k}}^{a}}
$$

where $m_{C O S_{k}}$ is the mass of $C O S_{k}$.

MEV can be equated to the "price" the exploration agent is willing to pay for a given unit of resource. In practice, MEV is found using a forward differencing approach; it compares the simulated exploration values of a baseline $a$ - scenario (without resources included) and an enhanced $b$-scenario (with resources included) and dividing by the mass of the resource.

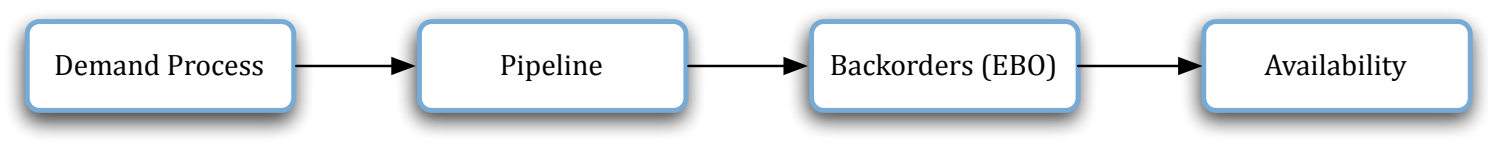

Figure 4-1: System availability estimation process 


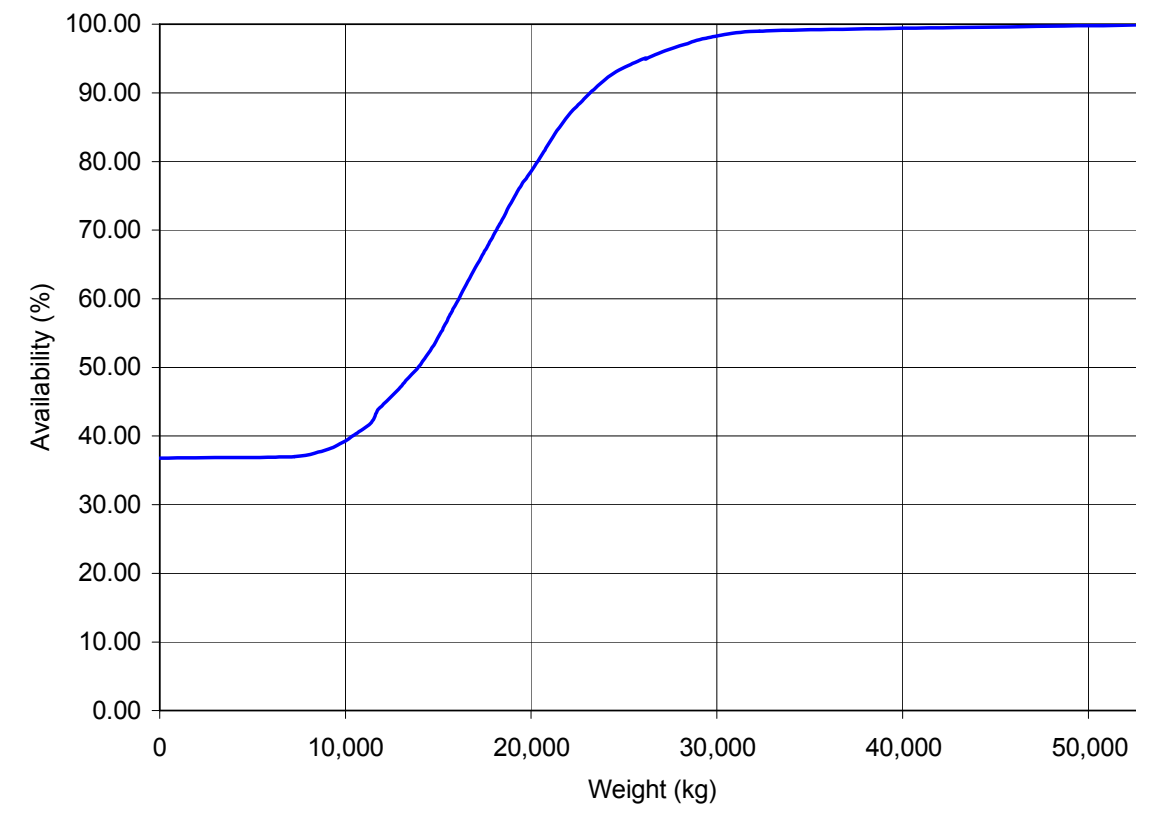

Figure 4-2: Availability-versus-resource curve for a nominal ORU in a simulated exploration system [54].

\subsubsection{Relative Exploration Value}

Finally, we define the Relative Exploration Value (REV) of a given basket of goods $b$ compared to another basket $a$ :

$$
R E V_{b, a}=\frac{E V_{t o t}^{b} / E V_{t o t}^{a}}{\prod_{j}\left(\frac{m_{C O S_{j}}^{b}}{m_{C O S_{j}}^{a}}\right)^{\beta_{j}}}
$$

where

$$
\beta_{j}=\frac{\omega_{j}^{a}+\omega_{j}^{b}}{2}
$$

given the following definitions:

$E V_{\text {tot }}=$ exploration value of a given basket

$m_{C O S_{j}}=$ total mass of class $j$ contained in a given basket

$\omega_{j}=$ mass fraction of $\operatorname{CoS} j$ in a given basket

REV is a divisia index, or a commonly computed linked index, which is designed to measure changes in productivity due to technology [55], and thus allows for consistent comparison of different baskets of resources in terms of exploration value. In the context of this thesis, REV is key in comparing the exploration value of different manifests.

\subsubsection{Defining the Available Resource}

The total cargo capacity mass (volume) of a parent element is represented by the large square. The rectangles to the right of the dark gray box represent the total mass of a nested element. The components include the dry mass of the nested element, the attach fittings/adapter mass required to nest the element on the parent, the accommodation mass (racks/lockers) required to hold cargo, 
the cargo in the nested element, and the stowage and restraint (packaging) mass used to pack the cargo. The parent element can also hold cargo itself, represented by the two rectangular boxes on top of the nested elements. Subtracting all of these components from the total cargo capacity yields the available cargo resource, to which revenue management may be applied.

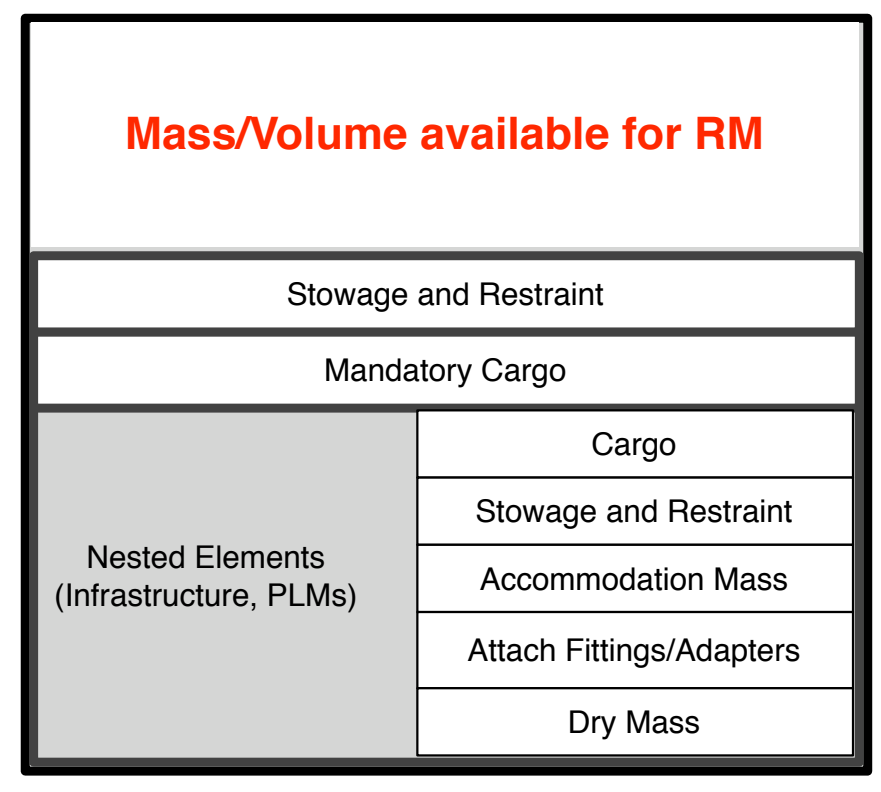

Figure 4-3: Available cargo resource for application of revenue management

\subsection{EMEV Algorithm}

The resource pricing module (RPM) estimates the MEV of a given supply item (or basket of supply items). Protection levels are found using Algorithm 1, which is based on an algorithm provided by Talluri and van Ryzin [10]. The idea is to simulate a large number, $K$, of demand vectors from the forecast demand distributions of each class over a given time horizon ${ }^{1}$. The next step is to progressively sort these values and partition the simulated demands in order to satisfy the above equation. The algorithm can be executed in $\mathrm{O}(\mathrm{nK} \log \mathrm{n})$ time, where $\mathrm{K}$ is the number of simulated vectors (reflecting the variance of the demand model). SpaceNet's existing high-speed simulation capability in conjunction with the good algorithmic performance of this approach make it an attractive option.

Once again recall the demand forecast in Table 3.1 in Section 3.2.1. The following sections will use this example to demonstrate how the EMEV algorithm works.

\subsubsection{Estimating Prices}

Recall from Section 4.1.2 that marginal exploration value (MEV) is defined as the marginal contribution of a given supply item to exploration value. However, in space logistics, the MEV of a given

\footnotetext{
${ }^{1}$ Typically this time horizon will capture a single sortie or flight. However, multi-flight horizons may be used. In either case, the time horizon should represent the upper bound of the duration of the mission since the EMEV algorithm presented will try to maximize performance within the bound.
} 


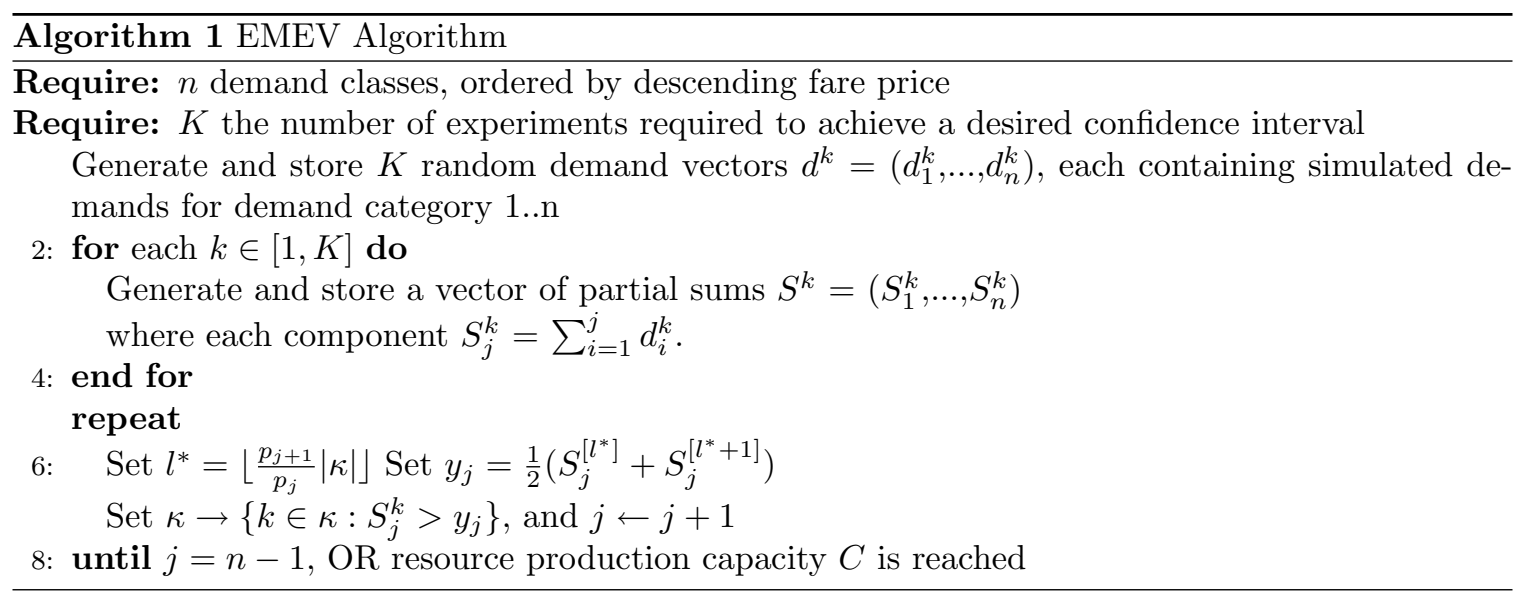

supply item is dependent on its position in the pipeline or sequence of supply items in its class that have already been manifested. This is because the value of a supply item is partially determined by the probability it will be used in the given time horizon, which is of course dependent on how many similar items have been manifested already. In terms of the airline analogy, it would be equivalent to the second passenger in a given booking class being charged a different amount than the first customer in that booking class.

To account for this in the pricing of each supply class, we consider the deterministic demand case, which will require $T_{j}$ supply item units to fully meet the demand for class $j$ over the user specified time horizon. The nominal price of a given class is approximated by taking the expected value of the MEVs over all $T_{j}$ supply item units as shown in in Equation 4.12. Once a price has determined for each class, prices are normalized so that the lowest fare class $p_{n}$ is equal to 1.0. Note that this does not change the output of the Algorithm 1, because it only depends only on the ratio of prices and not absolute prices (see Step 4). Normalization allows for more consistent comparison across scenarios and enables the user to more easily analyze pricing results.

$$
p_{j}=E\left[M E V_{j}\right]=\frac{1}{T} \sum_{t=1}^{T} M E V_{j, t}
$$

where $T$ is the number of supply items in class $j$ required in the deterministic case.

\subsubsection{Generating Demands and Partial Sums}

Each element $S_{j}^{k}$ of the demand vector $S^{k}$ is the estimated aggregate demand of each variable over the entire period. The number of demand vectors needed, i.e. the size of K, is selected to ensure a $95 \%$ confidence interval for each variable.

$$
K_{j}=\frac{s n}{2}\left(1+\operatorname{erf} \frac{x-\mu}{\sigma \sqrt{2}}\right)
$$

where $\mu$ and $\sigma$ are the mean and the standard deviation, respectively, of the demand generated by the nth variable over the time horizon. To be conservative, $K$ is set equal to the largest $K_{j}$.

SpaceNet analytically calculates the means and standard deviations for a sum of exponential, normal, and uniform distributions. For other distributions, the mean and standard deviation of a sum of a series random variables can be approximated using Lyapunov's Central Limit Theorem 
(LCLT). The LCLT which states that the sum of a large number of independent random variables will be approximately normally distributed if the random variables have a finite variance. The LCLT varies from the standard central limit theorem (CLT) in that it does not require a large number of identical random variables, but can rather handle a mix of variables. This enables more scalability and allows for interleaving processes as well as arbitrary event generation schemes. And indeed empirical evidence shows that most distributions converge to the normal distribution with a relatively small number of variables [56]. The implication of the LCLT is that for large number of different variables of arbitrary distribution, we can estimate the distribution of their sum as a normal distribution with $\mu$ and $\sigma^{2}$ as follows:

$$
\begin{gathered}
\mu=\sum_{n=1}^{N} \mu_{n} \\
\sigma^{2}=\sum_{n=1}^{N} \sigma_{n}^{2}
\end{gathered}
$$

Once the number of experiments needed to meet a specific confidence interval, $K$, has been determined, the corresponding number of experiments is generated by cloning the prototype experiment provided by the user and running a series of independent stochastic simulations. From these experiments, the set of demand vectors is formed as shown in Figure 4-4. For each demand vector $d^{k}$, a corresponding partial sum vector is formed. The partial sum vector is simply a running aggregate of the demands from the lowest class to the highest according to Equation 4.14. Figure 4-4 and Figure 4-5 for the general case and the quantitate three-class example, respectively.

$$
S_{j}^{k}=\sum_{i=1}^{j} d_{i}^{k}
$$

\subsubsection{Finding Optimal Protection Levels}

Talluri and van Ryzin prove that given continuous demand, a necessary and sufficient condition for $y *$ to be an optimal vector of protection levels is that it satisfy the $n-1$ equations:

$$
P\left(B_{j}\left(y^{*}, D\right)\right)=\frac{p_{j+1}}{p_{1}}, j=1,2, \ldots, n-1
$$

where $B_{j}\left(y^{*}, D\right)$ is the event that the demand to come in future stages exceeds the corresponding protection levels and $D=D_{1}, \ldots, D_{n}$ is a vector of demands for each class

The above says that the probability that the $j^{\text {th }}$ demand will be realized is equal the ratio of class $(j+1)$ revenue to class 1 revenue; this essentially reduces to Littlewood's rule in the $n=2$ case. Though this method is suited to continuous demand, it provides a good approximation for discrete demand as well. Building on Equation (4.15), it follows that the protection levels between sequential classes should reflect the ratio of their prices:

$$
P\left(B_{j}\left(y^{*}, D\right)\right)=P\left(D_{1}+\ldots+D_{j}>y_{j} \mid B_{j-1}(y, D)\right) P\left(B_{j-1}\left(y^{*}, D\right)\right)
$$




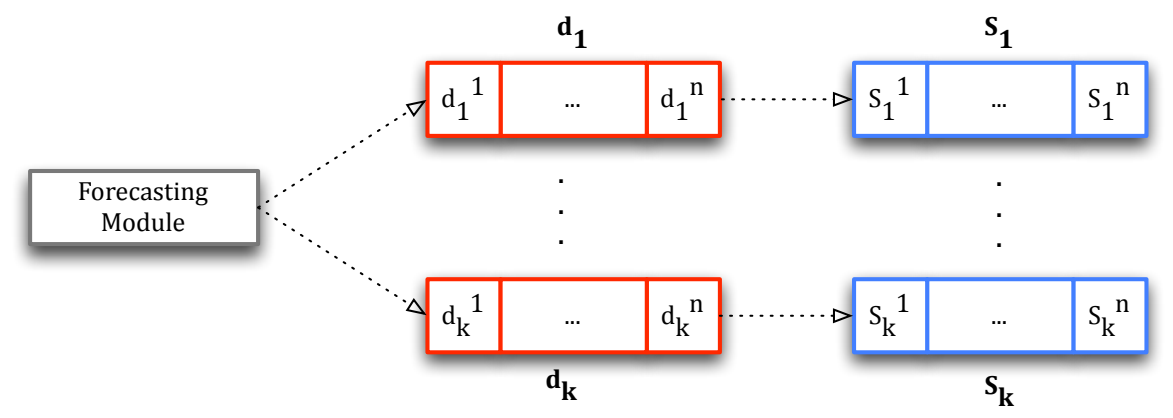

Figure 4-4: Demand vector $\left(d_{k}\right)$ and partial sum vector $\left(S_{k}\right)$ generation - general case

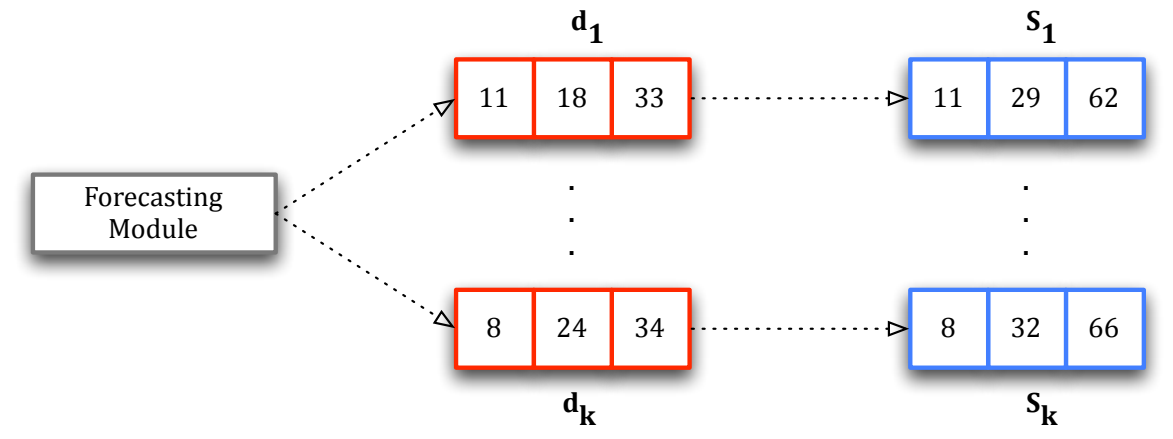

Figure 4-5: Demand vector $\left(d_{k}\right)$ and partial sum vector $\left(S_{k}\right)$ generation - quantitative example based on Table 3.2

which implies that the optimal $y^{*}$ must satisfy:

$$
\begin{aligned}
P\left(D_{1}+\ldots+D_{j}>y_{j}^{*} \mid B_{j-1}(y, D)\right) & =\frac{1}{P\left(B_{j}\left(y^{*}, D\right)\right)} \frac{p_{j+1}}{p_{1}} \\
P\left(S_{j}>y_{j}^{*} \mid B_{j-1}(y, D)\right) & =\frac{p_{j+1} 2}{p_{j}} .
\end{aligned}
$$

where $S_{j}=D_{1}+\ldots+D_{j}=\sum_{i=1}^{j} D_{i}$.

Iterating through sequential classes in a high fare to low fare order establishes a series of protection levels for each class. The last class (i.e. the lowest fare class) is allocated any capacity above the penultimate class' allocation.

Consider our favorite 3 three class example. From Table 3.2, the price ratios between the classes are found to be $p_{2} / p_{1}=70 / 100=0.7$ and $p_{3} / p_{2}=42 / 70=0.6$. The first iteration of the above algorithm finds a protection level for the first class, $y_{1}$, such that $70 \%$ of the points have $S_{1}$ values above $y_{1}$ (since $p_{2} / p_{1}=0.7$ ). In the next iteration, the points to the right of the first partition are sorted by the $S_{2}$ values. The class 2 protection level, $y_{2}$, is chosen such that $60 \%$ of the points lie above it (since $p_{3} / p_{2}=0.6$ ). Thus the protection levels $y_{1}^{*}$ and $y_{2}^{*}$ have been found, with any remaining capacity above $y_{2}^{*}$ being allocated to class 3 . 


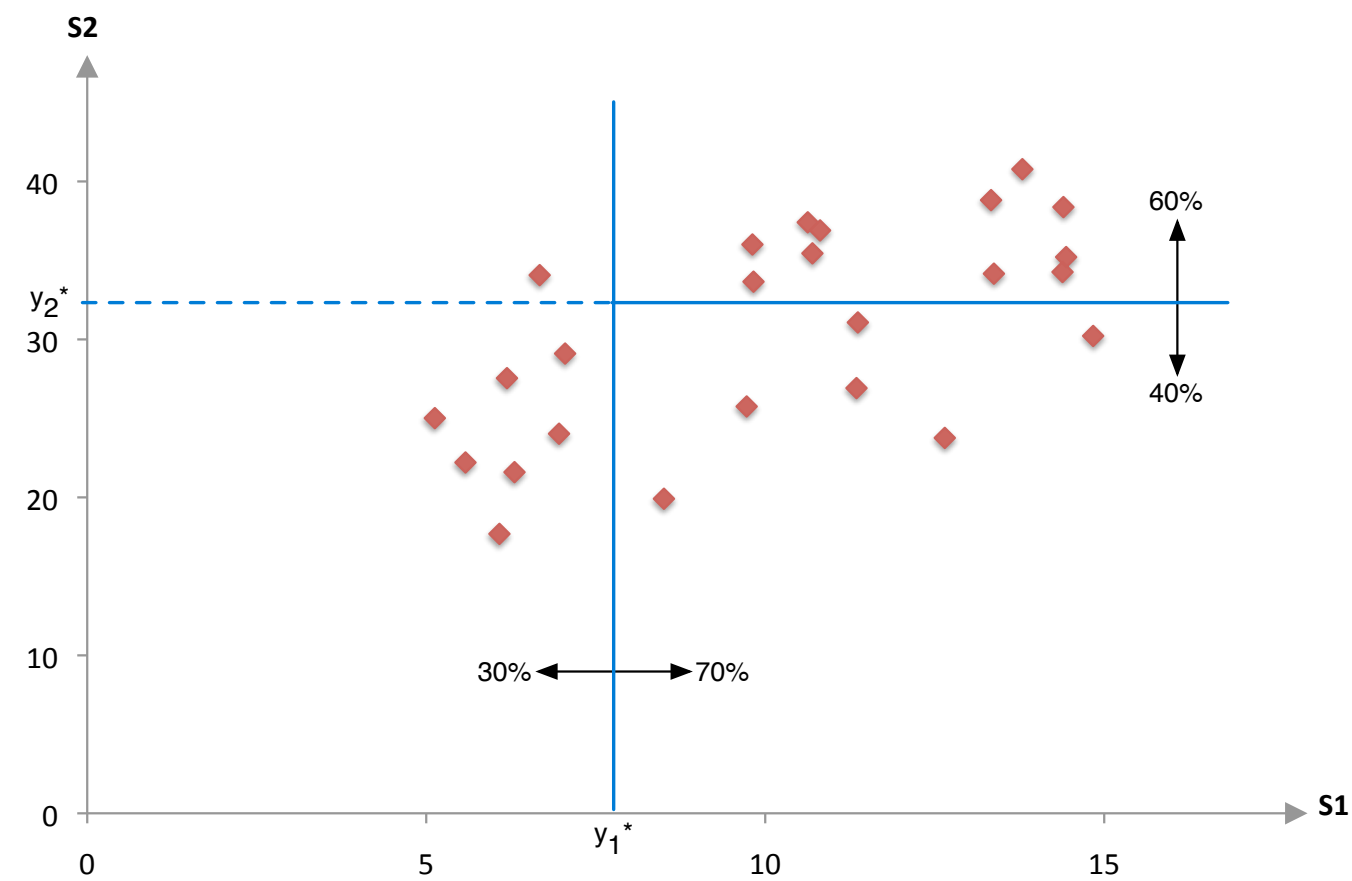

Figure 4-6: Plot of the partial sums S1, S2 and the resulting Monte Carlo estimates of optimal protection levels for 25 simulated data points

\subsection{Greedy Bid-Price Algorithm}

The second algorithm for determining protection levels is based on self-adjusting bid-prices. Selfadjusting bid-prices differ from the conventional bid-prices by because they are recomputed for each resource request. The benefit of self-adjusting bid prices are that they avoid using outdated information to make capacity decisions. However, because of the frequency with which they recalculate the opportunity cost function, they must generally use relatively simple parametric value functions that are computationally inexpensive. In this specific case, the value function is MEV.

Algorithm 2 extends the greedy algorithm that Rinnooy-Kan, et al. present for the multidimensional knapsack problem [57], and modifies it for this application. First, the marginal exploration value for each fare class is calculated according to Equation 4.9 (Line 3). Next the bid-price equal to the maximum MEV value (Line 5). Finally an additional unit of any class that exceeds the bid-price is manifested. Since the bid-price is set to the highest MEV value, essentially this algorithm is greedily selecting and manifesting the most profitable class in each iteration. This cycle repeats until the production capacity of the resource is reached, and the final manifest provides the protection level for each class.

\subsection{SpaceNet RM Implemenation}

The integrated modeling and simulation capability provided by SpaceNet is a solid foundation upon which to implement a revenue management system for space exploration. However, it was still necessary to develop a suitable definition and metric for revenue and to design and implement 


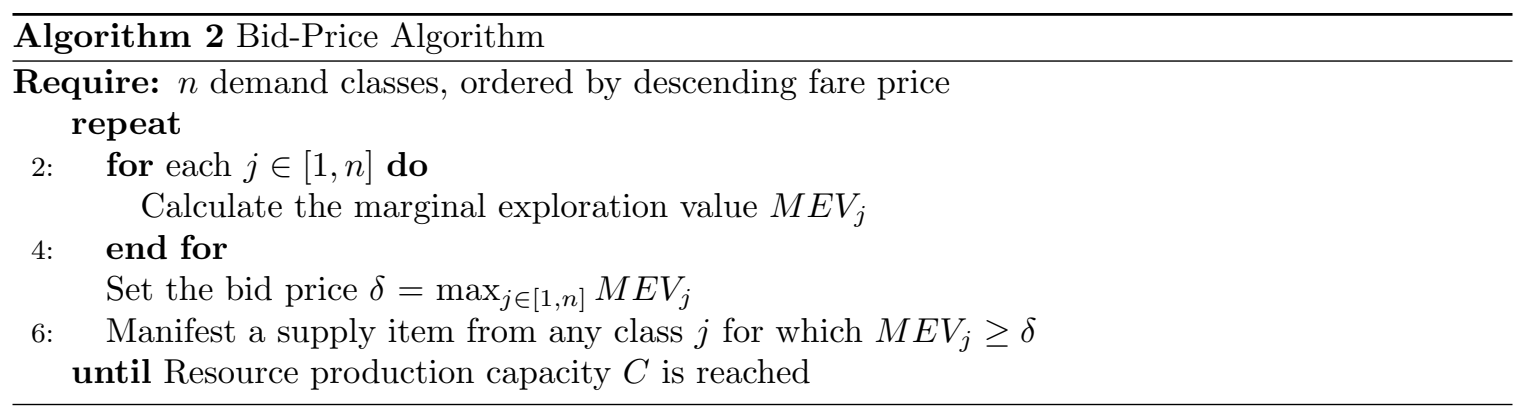

several new features. These were bundled into SpaceNet RM, a new plugin module available for SpaceNet 2.1 and beyond.

Figure 4-7 shows the components of the SpaceNet RM plugin, its relationship to the core SpaceNet modules, its required inputs, and finally its outputs. SpaceNet RM uses SpaceNet 2.0's Forecasting Module to estimate demand and the appropriate level of supply to meet a target availability. This information is fed into the Pricing Module which estimates the expected revenue of each supply item using MEV. The Manifesting Module uses the Greedy Bid-Price heuristic and the prices generated by the Pricing Module, to "optimally" manifest resources in simulation. The Capacity Allocation Module uses the EMEV heuristic and the prices from the Pricing Module to provide "optimal" capacity allocations for a given exploration architecture.

\subsection{Chapter Summary}

This chapter presented the conceptual framework of SpaceNet revenue management, and the implementation of two control algorithms, EMEV and Greedy Bid-Price, that can be used to provide capacity allocation estimates for SpaceNet scenarios. The primary contribution of the conceptual framework was to introduce marginal exploration value (MEV) as a proxy for monetary revenue. Marginal exploration value is essentially a probabilistic extension of exploration capability (EC); it reflects the exploration capability and the probability that that capability will be realized. The EMEV algorithm is a Monte Carlo integration based approach that utilizes the expected marginal exploration value of each class to segment available capacity by class. The bid-price algorithm is a greedy algorithm that assigns capacity based on choosing the consumer that provides the maximum EMEV at any given time.

Chapter 5 details some analysis performed on campaigns of lunar missions using SpaceNet. 


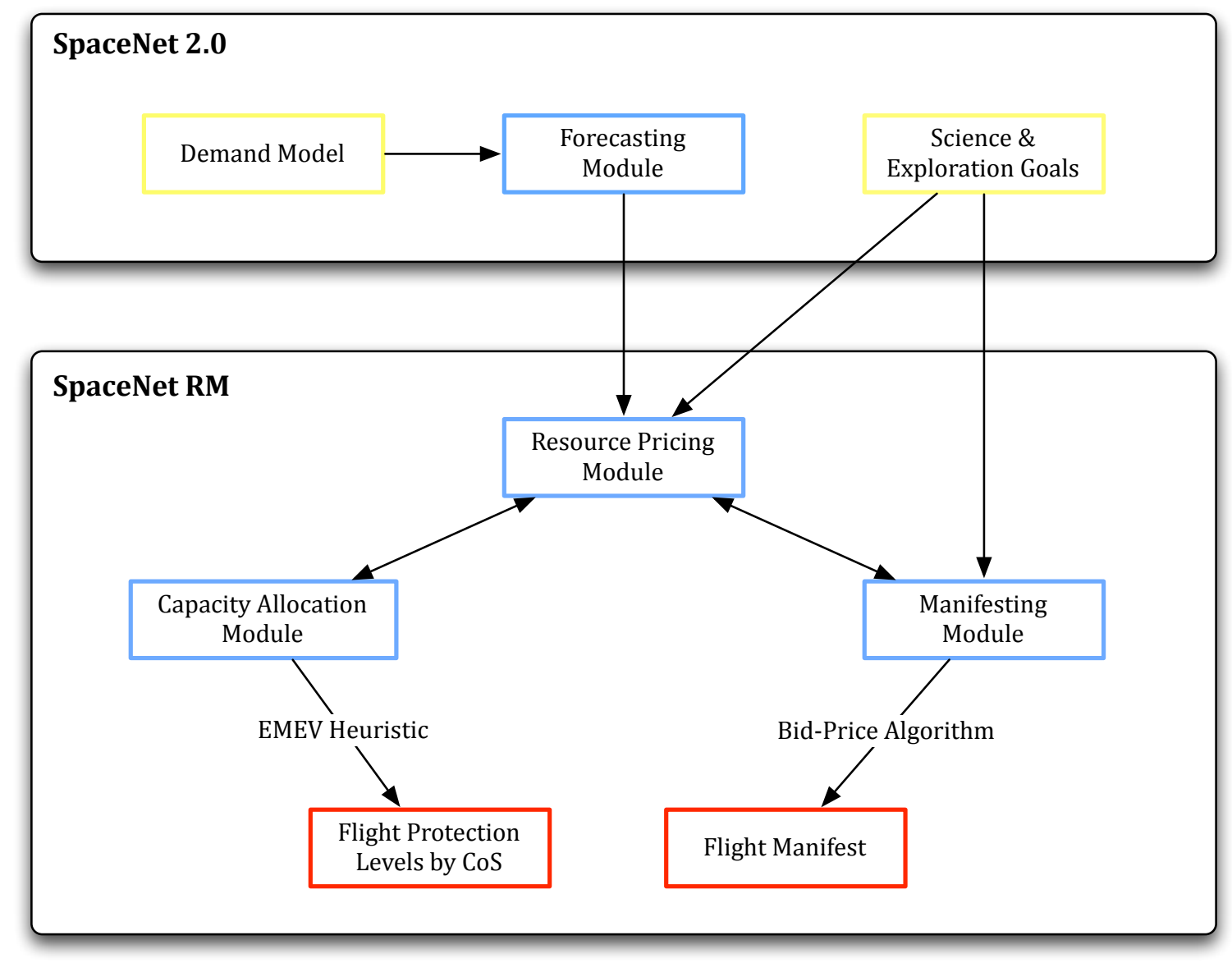

Figure 4-7: Spacenet RM implementation structure 


\section{Chapter 5}

\section{Results and Conclusions}

\subsection{Overview}

The Constellation program has a stated goal of global exploration of the Moon while achieving a continuous presence. To the end of global exploration, the 2006 ESAS report identified ten candidate lunar sites for exploration on the basis of operational, scientific, resource potential, and programmatic considerations [4]. These were the Lunar South Pole, SPA Basin Floor, Orientale Basin Floor, Oceanus Procellarum, Mare Tranquillitatis, Smythii Basin Floor, Central Far Side Highlands, North Pole, Aristarchus Plateau, and Rima Bode. In general, these sites are preferred because they offer many different features that cumulatively satisfy a wide variety of technical and scientific interests. They offer geological diversity for science as well as high-grade ore bodies to maximize ISRU production while minimizing the investment of time and energy.

The mobile outpost mission type provides an interesting option for providing both the depth and the breadth of exploration that Constellation requires. In addition, of the mission types discussed in Section 1.2.1, the mobile outpost provides an ideal test for the application of revenue management because it features both demand heterogeneity due to the various infrastructure elements and the crew, and limited operational flexibility due to the relatively minimal storage and transfer of consumables between sites. The following sections focus on the application of the SpaceNet RM framework to the high-level evaluation of the mobile outpost logistics strategy.

\subsection{Winnebagos and Jeeps}

One emergent mobile base concept is the "Winnebagos and Jeeps" concept described by Wilcox [58]. In this concept, mobile habitats and laboratories are used as "Winnebagos" for long-range exploration, storing supplies and consumables while providing the power and life support functions that enable long-term, extended-range exploration. Supporting the Winnebagos are long-range pressurized rovers, or "Jeeps", that allow astronauts to quickly and easily reach sites of scientific interest, putting human reasoning and intelligence at the forefront of sample-gathering activities. Jeeps also support the transportation of crew, and their provisions from site to site. According to Wilcox, one winnebago and jeep pair could potentially explore thousands of kilometers, and two pairs would likely be enough to provide global-scale exploration [58].

To demonstrate the strategy, Wilcox developed an exploration plan to visit eight of the ten lunar 
Table 5.1: Winnebagos and Jeeps mobile outpost exploration detailed plan

\begin{tabular}{|l|c|c|c|}
\hline ESAS Site & $\begin{array}{c}\text { Distance from } \\
\text { previous site }(\mathrm{km})\end{array}$ & $\begin{array}{c}\text { Travel time from } \\
\text { previous site (months) }\end{array}$ & $\begin{array}{c}\text { Lander } \\
\text { capability }(\mathrm{mT})\end{array}$ \\
\hline 1. South Pole & 0 & 0.0 & 6.0 \\
2. SPA Basin Floor & 1400 & 2.3 & 4.0 \\
3. Orientale Basin Floor & 2600 & 4.3 & 5.0 \\
4. Oceanus Procellarum & 1800 & 3.0 & 4.0 \\
5. Mare Tranquillitatis & 2000 & 3.3 & 4.0 \\
6. Smythii Basin Floor & 2400 & 4.0 & 4.0 \\
7. Central Far Side Highlands & 3000 & 5.0 & 5.0 \\
8. North Pole & 2500 & 4.2 & 6.0 \\
\hline
\end{tabular}

sites identified in the ESAS report, as summarized in Table 5.1 and Figure 5-1. In this scenario, a dedicated cargo flight would deliver large infrastructure items and the first scientific payload to the first site (South Pole) ahead of any crewed missions. The site would be robotically scouted and various mission systems checked out remotely. Following this, the tour of the eight target exploration sites would begin, with the following sequence of events occurring at each site:

1. Crew lands near to pre-positioned mobile infrastructure.

2. Crew unloads any consumables and exploration equipment, and performs any necessary assembly tasks.

3. Using Jeeps, the crew begins high-intensity exploration of the extended exploration site area.

4. The crew uses a pre-positioned ascent stage to return home.

5. Mobile infrastructure departs for the next site using a combination of autonomous control and teleoperation.

Designed correctly, mobile habitats can avoid the duplication of structure, thermal control, power, communications, and navigation equipment required for two separate vehicles, thereby offsetting the cost of transporting their additional mass around the lunar surface. Additionally, the flexibility provided by the rovers allows new exploration paradigms that are more capable of global exploration, while providing a depth of exploration similar to that of an outpost. For instance, the crew may use the winnebago as a stationary camper, utilizing the rover to travel up to $200 \mathrm{~km}$ away from the mobile habitat and only returning periodically to resupply and explore new directions. Alternatively, the mobile habitats may be used as a true winnebago, moving in tow behind the jeep and allowing the crew ultimate freedom to explore for an extended period of time, only limited by the amount of cargo that can be carried along. From the above description, one can imagine that this architecture uses a mixture of carry along, pre-positioning, and resupply flights.

\subsection{Experimental Setup}

As a case study, consider a two winnebago plus two jeep scenario based on the Constellation lunar architecture. Constellation nominally requires 4 crew members per mission, as well as anytime return through a dedicated ascent stage. Thus, the following items, or customers, are on the surface for each mission: 


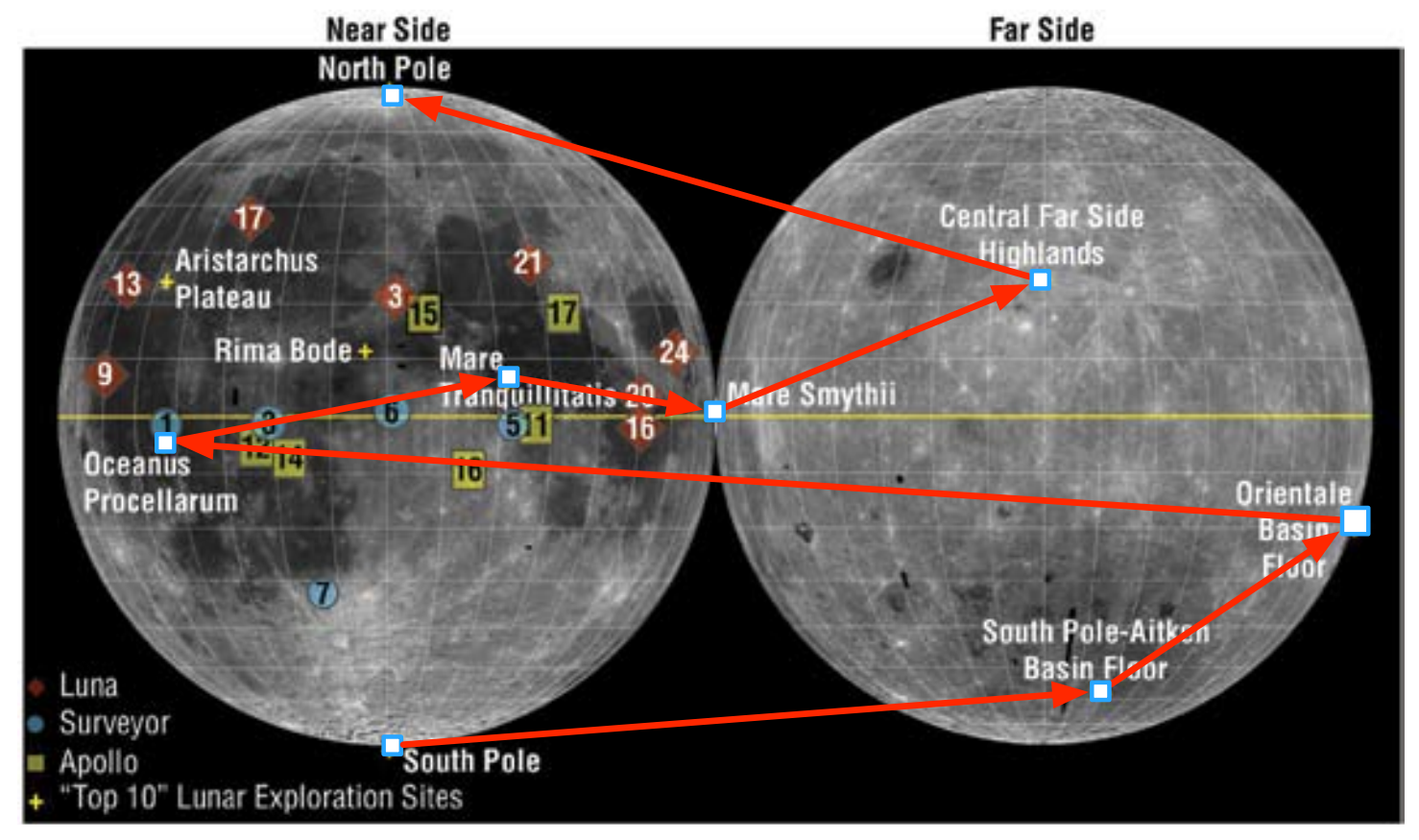

Figure 5-1: Winnebagos and Jeeps mobile outpost exploration concept

- Agents: Four human explorers

- Mobile Lab: The first winnebago; storage center for operational supplies and storing consumables from site to site. Also includes power generation, drilling, and excavation equipment.

- Sortie Hab: The second winnebego. Contains living quarters of the crew, and includes ECLSS system and communications terminal.

- Pressurized Rover (x2): The jeeps in this scenario. The pressurized rovers feature a $200 \mathrm{~km}$ range, enabling the astronauts to travel up to $100 \mathrm{~km}$ away from the central site.

Note that the above model does not account for the descent or ascent modules (i.e. the transportation elements) in the demand model.

Each customer produces demands based on the demand model in Table 5.2. Using the fact that a series of exponential inter-arrivals times can be modeled as a Poisson process, and a sum of series of Poisson processes is itself a Poisson process with a rate equal to the sum of the rates of the individual processes, we can simplify the spares demand significantly. Note that nominal rates are based off LAT data and Larson \& Pranke [59].

For simplicity, the detailed and complex accommodation, attach fittings and adapters, and pressurized carrier nesting structure has been abstracted to a simple tare factor, which represents the amount of mass associated with those items for each unit of mass of actual supply item manifested. 
Table 5.2: Demand model for Winnebagos and Jeeps mobile outpost scenario

\begin{tabular}{|l|c|c|c|l|}
\hline Variable & Distribution & Mean (units) & Standard Dev. & Tare Factor \\
\hline Crew Provisions & Normal & $2.75(\mathrm{~kg} / \mathrm{crew} /$ day $)$ & 1.07 & $120 \%$ \\
Crew Operations & Normal & $1.0(\mathrm{~kg} / \mathrm{crew} /$ day $)$ & 0.3 & $100 \%$ \\
Pressurized Spares & Poisson & 0.06 (failures/day) & 0.06 & $80 \%$ \\
Unpressurized Spares & Poisson & 0.03 (failures/day) & 0.03 & $100 \%$ \\
\hline
\end{tabular}

\subsection{Experimental Results}

The following sections investigate the effect of various parameters on EMEV, and subsequently capacity allocations between the demand classes. Section 5.4.1 and Section 5.4.2 provide some intuition about the effect of delivery capability, mission duration, and spares demands, respectively. Section 5.4.3 discusses the capacity allocations for the entire mobile outpost scenario based on simulation.

\subsubsection{Mission Duration}

Just as delivery capability has an effect on the capacity allocation, the nominal duration of a mission also has an effect on capacity allocation.

Figure 5-2 and Figure 5-3 show the evolution of the MEV of each supply class in Table 5.2 with respect to mission duration (i.e. the length of the time horizon provided by the user) using the EMEV and Greedy Bid-Price algorithms, respectively. From the figures, it is evident that while the MEVs of the operations and crew provisions classes are relatively constant over with respect to mission duration, the spares MEV shows much more dynamic behavior. For very short mission lengths, the MEV of spares is low, reflecting the fact that any breakdown is unlikely in a short mission. As the mission duration increases, the spares MEV increases to reflect the increasing need for spares. The spares MEV reaches a peak at about 30 days, after which it falls gradually and almost monotonically (the stochastic nature of the algorithm leads to small deviations from monotonicity), eventually settling down to a constant value by about 180 days. This constant value represents the deterministic contribution of a spare that is frequently used in spare-by-mass models. The peaking behavior is due to the underlying summation of several Poisson random variables with different interval values that is used to estimate MEV (see Equation 4.12).

The results show that using deterministic models to set capacity allocations may be sufficient for longer missions, but those models significantly underprice the value of spares (and therefore its capacity allocation) in shorter missions. Thus it is important to recalculated the capacity allocations for each class of supply as mission durations change significantly (typically caused by reduced vehicle performance or modified demand models), rather than simply scaling down the long duration allocations.

\subsubsection{Spares Uncertainty}

Not surprisingly, spares uncertainty plays a large part in logistical performance. While systems are thoroughly tested on Earth, in-space environmental and operational factors lead subsystems to experience different (usually lower) levels of performance in actual use. This results in a changed spares demand rate, a different valuation of spares, and subsequently a different optimal capacity allocation. To capture this effect, we define the K-factor as a failure rate multiplier that integrates 


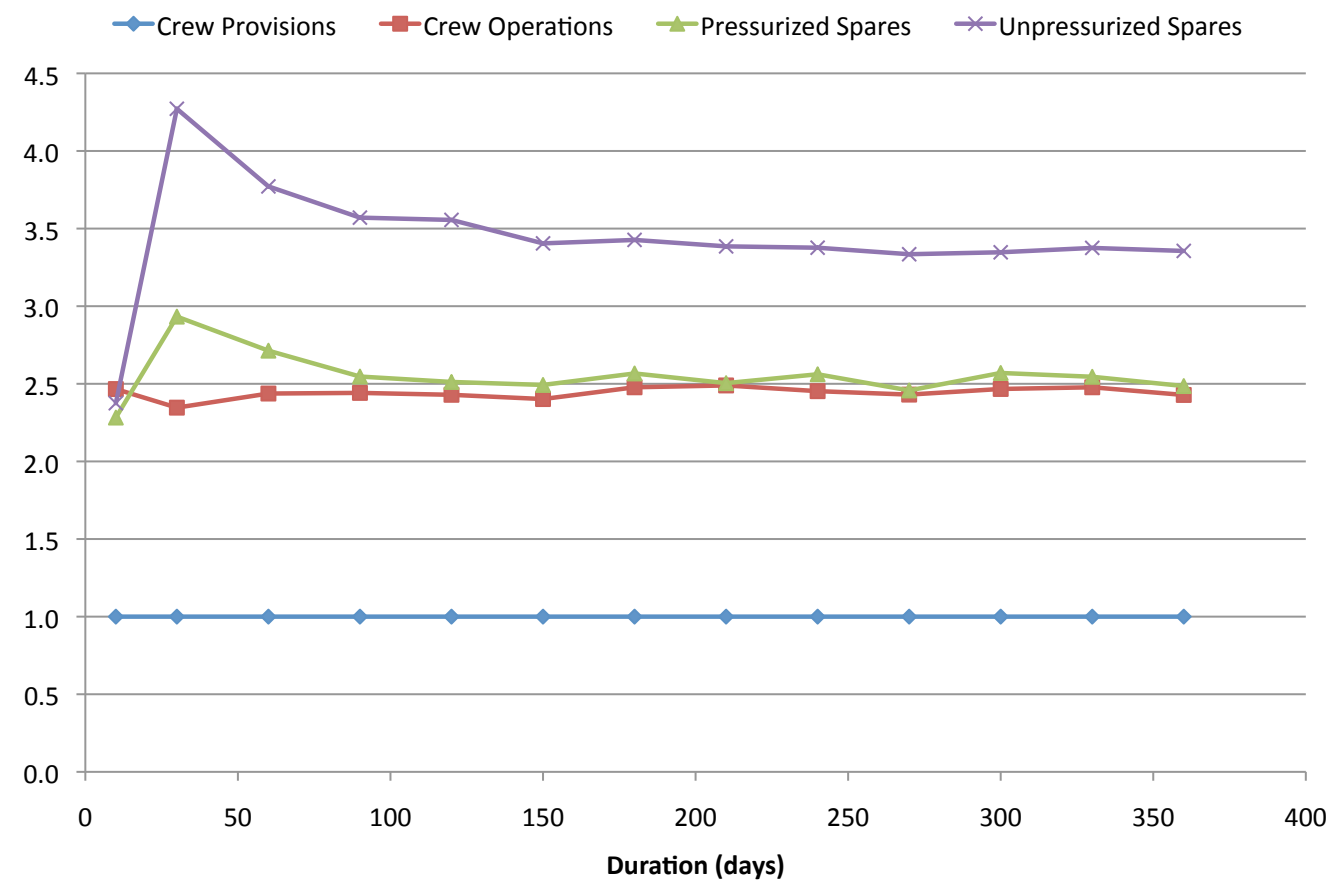

Figure 5-2: Normalized MEVs versus mission duration using EMEV algorithm

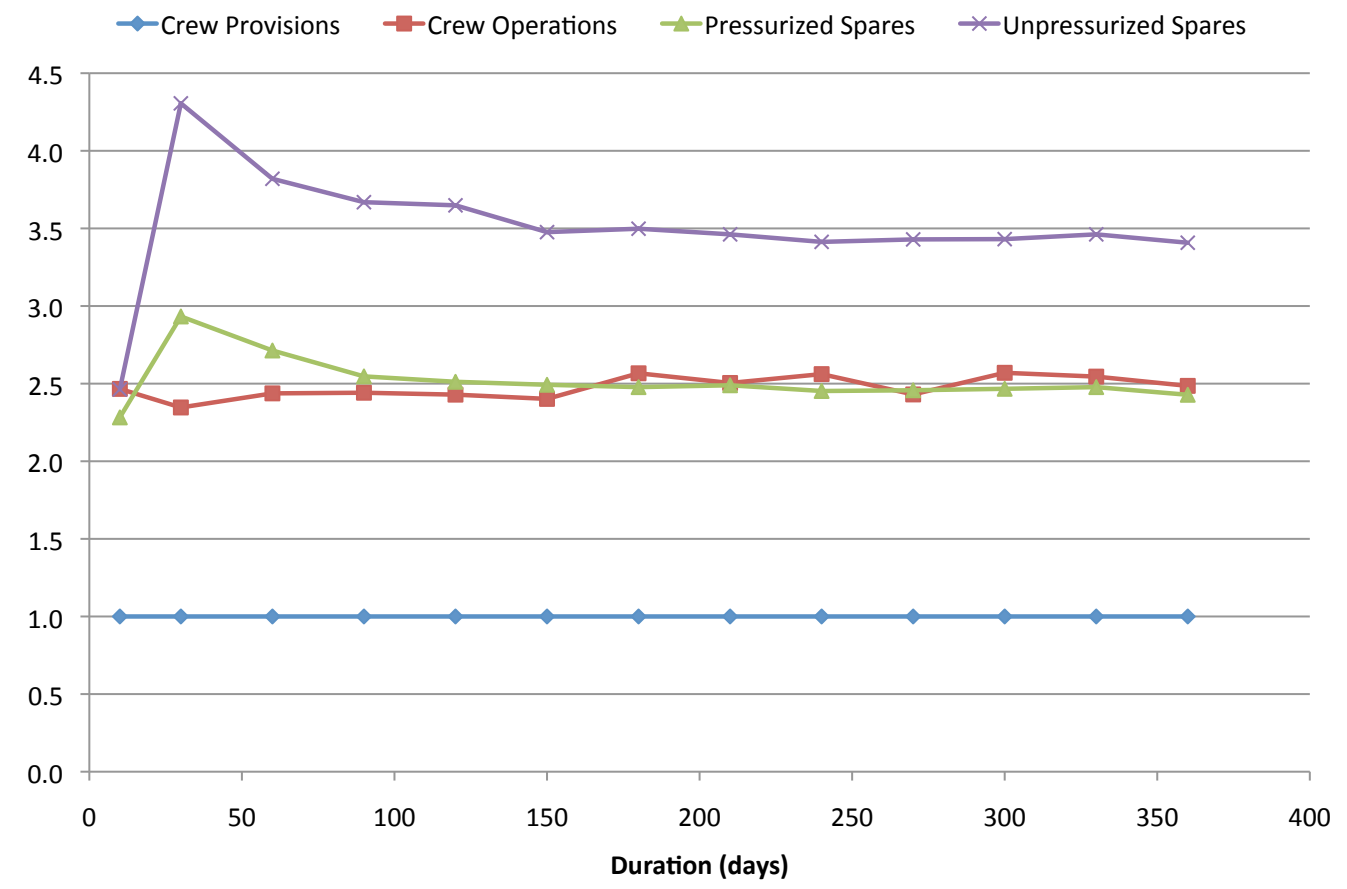

Figure 5-3: Normalized MEVs versus mission duration using Greedy-Bid Price algorithm 


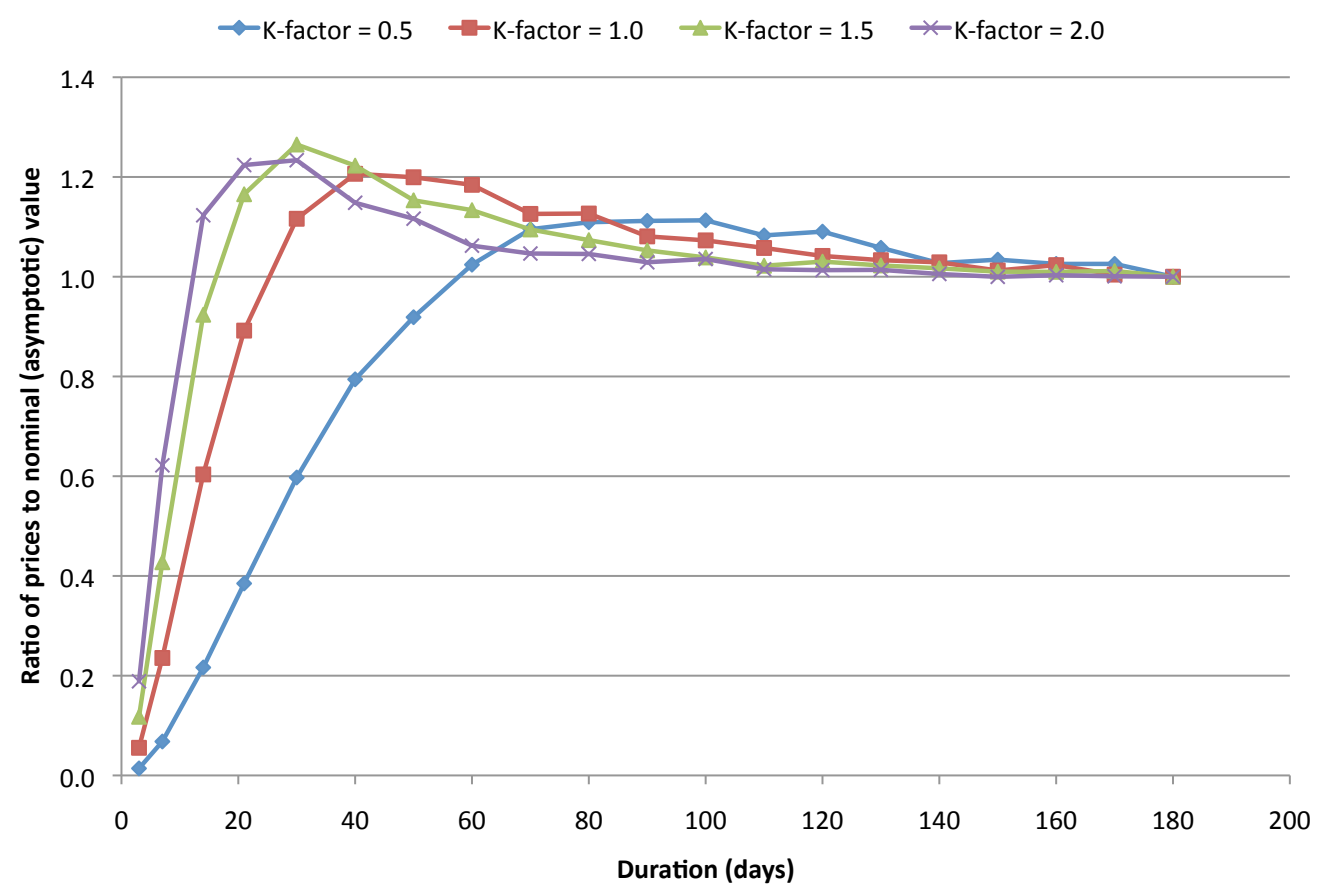

Figure 5-4: Evolution of MEV versus K-factor as a fraction of the deterministic case estimate

environmental factors and operational factors. The K-factor multiplies the nominal mean time between failure, $M B T F$, to produce an adjusted operational value, $M B T F^{\prime}$.

Figure 5-4 shows the effect of K-factor on the marginal exploration values for unpressurized spares using the EMEV algorithm. As K-factor grows, the demand for spares grows, resulting in two primary effects. First, an increased K-factor reduces the nominal MEV of a unit of spares relative to the other demand classes, and second it shifts the peak MEV of the spares class to an earlier date. Recalling that in Figure 5-2, in which the K-factor was 1.0, the peak MEV of spares was at about a 30 days. At a K-factor of 2 the peak shifts to about 10 days and at a K-factor of 0.5 , the peak shifts to about 40 days. In other words, the less frequent spares demands become, the more rapidly the MEV converges to its long term nominal value.

Recall from the experimental setup that a composite spare that represents the weighted average of all the many types of spares in a typical exploration system was derived to simplify the demand model. However, a real system may feature tens if not hundreds of types of spares, each with a different K-factor. The results here suggest that in additional to improving high level capacity allocation decisions, revenue management can also be used at a more detailed level to divide the global spares allocations optimally between the various types of spares, and adjust these allocations based on K-factor as more operational data becomes available.

\subsubsection{Mobile Outpost Protection Levels}

Recall once again the Winnebegos and Jeeps scenario described in Section 5.2. A series of experiments were run to determine what the evolution of protection levels would be at the mobile outpost given the travel plan shown in Table 5.1. The objective was to investigate how capacity allocations vary over the course of the campaign as different amounts of cargo are transferred between different 
sites, with each mission given a nominal duration of 30 days. For the transfer of cargo, it was assumed that the demand from the previous mission was exactly the mean value, meaning that the residual cargo was also the mean value.

Figure 5-5 and Figure 5-6 show the results of the first variation using the EMEV and bid-price algorithms, respectively. What the results show is that both algorithms initially give extremely generous allocations to unpressurized spares, the highest MEV class based on the demand model specified, before tapering off to the nominal values as the campaign progresses. While unpressurized spares dominate the early manifesting, pressurized spares are given preferential allocations through the mid-latitudes (Missions 4 through 7). Crew operations and crew provisions on the other hand are manifested at a fairly stead rate throughout the campaign, reflecting the relatively deterministic nature of their demand.

Intuitively, one can imagine that the capability of the lander effects the maximum duration of a mission, which in turn affects capacity allocations for the various demand classes. From Table 5.1, one can see that each site has a different nominal landing capacity. This is a result of the different orbits required to enable the lander to reach the site, and the correspondingly different amounts of $\Delta V$ (i.e. fuel) required to enter those orbits after translunar injection (TLI). These differences in lander capability, combined with the limited cargo storage capacity of a mobile outpost as compared to a stationary outpost, make exploring the mid-latitudes to the same degree as the poles especially difficult. The algorithms compensate for this phenomenon by ensuring availability in the most mass-

efficient way possible, which in this case means ensuring infrastructure availability through a buildup of both types of spares.

\subsection{Algorithm Comparison}

The results of the various experiments in Section 5.4 show that the EMEV and Greedy Bid- Price algorithms produce very similar manifests. This is not surprising since they use the same marginal exploration value (MEV) control. However, since EMEV is a continuous approximation of a discrete phenomenon, it tends to produce smoother output than Greedy Bid-Price. The tradeoff for the increased precision is computational time; EMEV is a Monte Carlo method that can require a large number of samples, particularly as one models spares demands in more detail. 


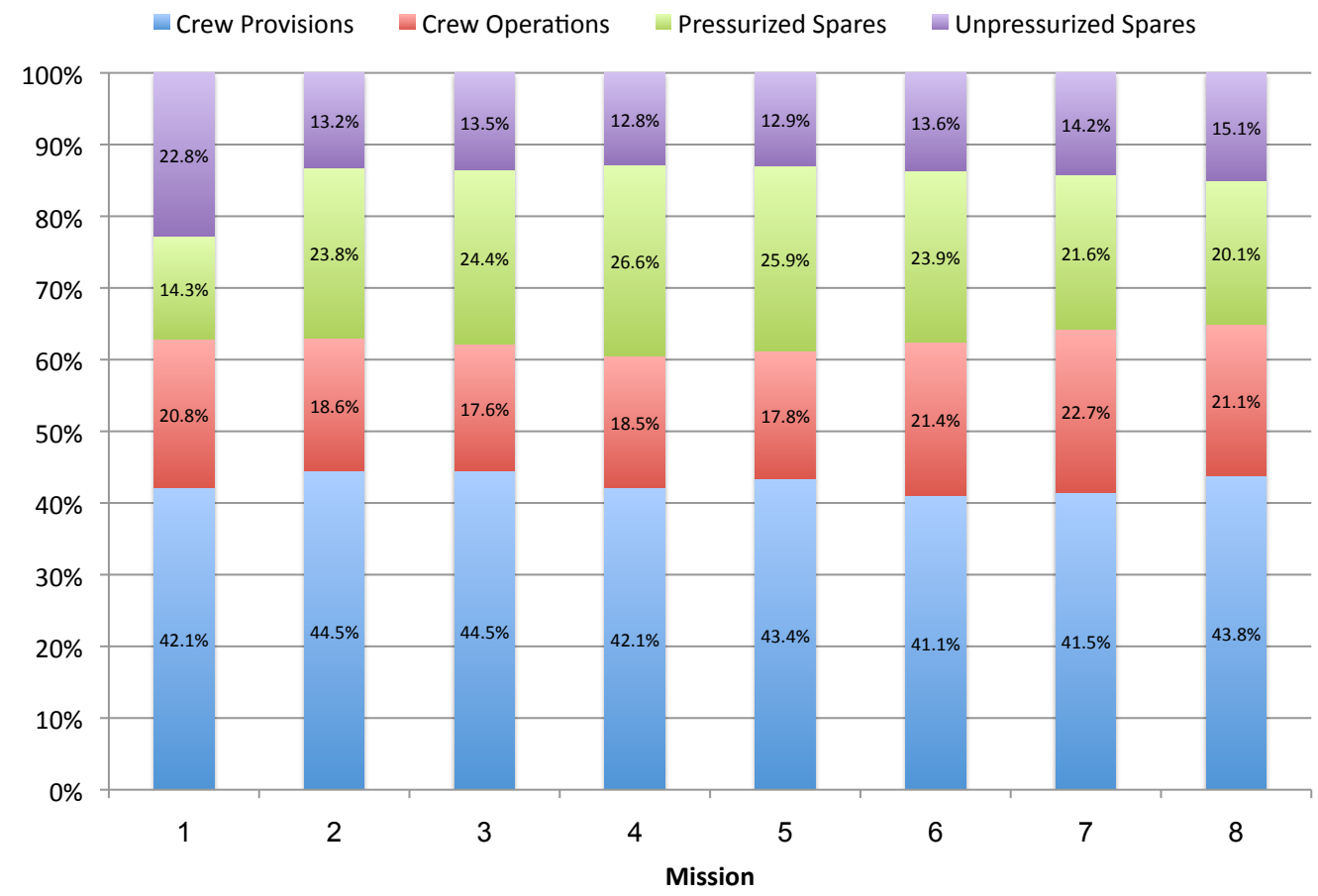

Figure 5-5: Mission-by-mission capacity allocations for Winnebagos and Jeeps mobile outpost scenario using the EMEV algorithm

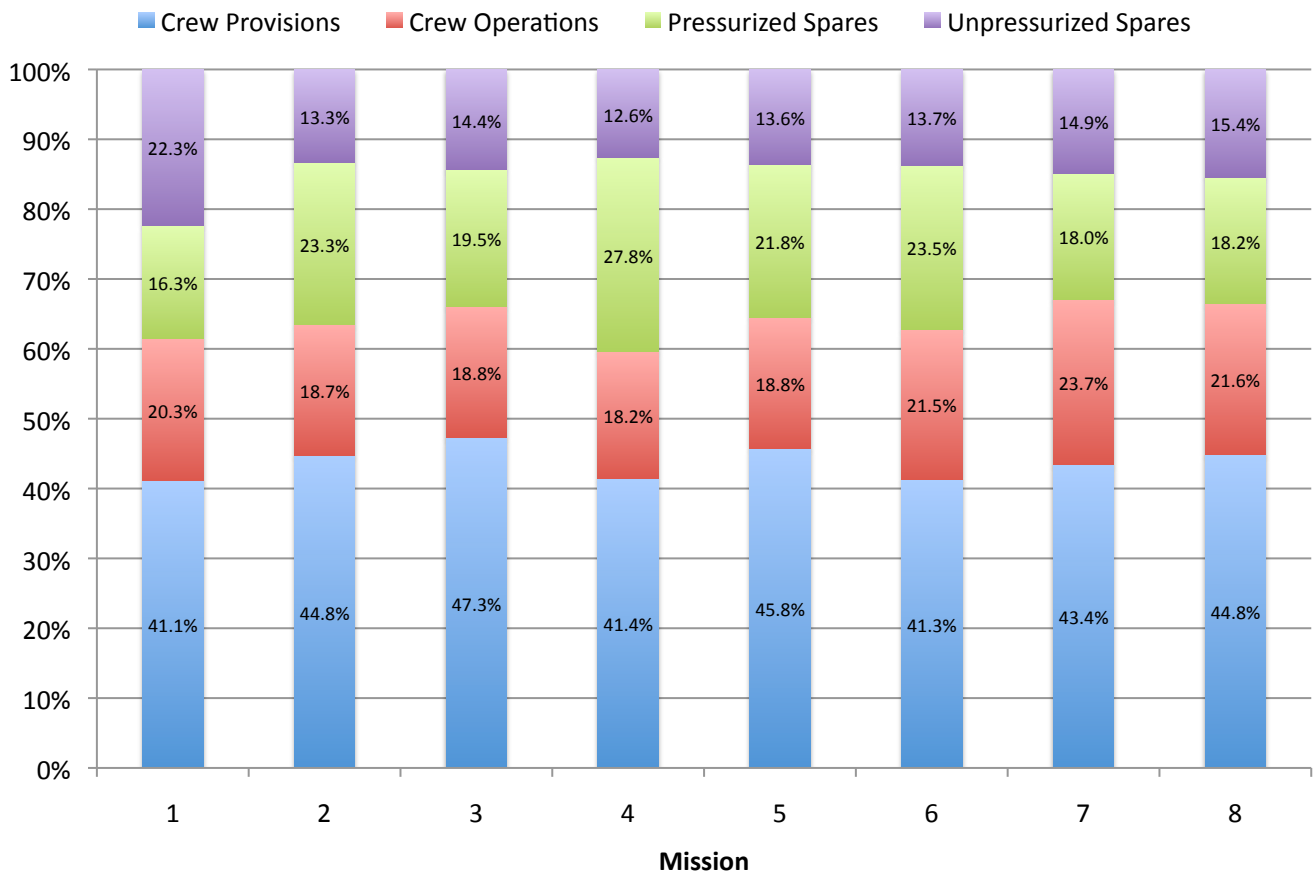

Figure 5-6: Mission-by-mission capacity allocations for Winnebagos and Jeeps mobile outpost scenario using the Greedy Bid-Price algorithm 


\section{Chapter 6}

\section{Conclusions}

This chapter presents the important conclusions that can be drawn from the work summarized in this thesis as well as potential areas of future work.

\subsection{Findings and Contributions}

Revenue management has significantly altered the travel and hospitality industry, and is now beginning to exert influence on a host of other businesses. At the core, revenue management uses detailed market knowledge and sophisticated controls to help capture additional incremental revenues from an existing capacity constrained resource. This makes space logistics, which is the design and analysis of cargo flow in space missions, an ideal candidate for revenue management.

The main contribution of this thesis is the application of revenue management to space logistics, including the development of a theoretical framework and the formulation and implementation of control algorithms. Specifically, the following contributions have been made:

- The categorization of space logistics as a capacity control problem with an exploration-based value function.

- The development of a value function, Marginal Exploration Value (MEV), as a proxy for the revenue of an exploration campaign

- The implementation of two heuristic controls - EMEV and Greedy Bid-Price - as part of the revenue management add-on module for SpaceNet 2.0 (SpaceNet RM).

Each of these findings will be contributions further in the following sections.

\section{Capacity Control}

The first important contribution is the identification of space logistics as fundamentally a capacity control problem. The goal of space logistics is to determine how best to allocate the resources to support exploration, which is closely aligned with the quantity-RM problem. Recall from Section 3.2.2 that dynamic pricing is not applicable because it requires demand to be endogenous to the producer's price control; in space logistics demand is exogenous to the price control. Overbooking is not particularly applicable because for all intents and purposes, capacity is fully utilized in space logistics (i.e. all the customers show); the question is how to ensure that each supply item in the 
manifest makes a nonzero contribution to exploration (i.e. the customer pays). Finally, estimation and forecasting are already handled in large part through the ISCMLA framework through demand models driven by the 10 major classes of supply.

\section{Marginal Exploration Value}

The second important contribution is the definition of marginal exploration value (MEV) as a proxy for revenue. Space logistics features many qualitative and quantitative components of revenue, few of which have anything to do with monetary amounts. In addition, exploration campaigns can develop safety stocks, which threatens one of the four basic conditions for the implementation of revenue management (see Section 1.3.1). MEV solves the first issue by defining marginal revenue as the marginal contribution to the ability to conduct exploration, thereby abstracting the complex web of interests that drive real exploration programs. Additionally, MEV prices new supply items based on the existing stockpile of supply items at the exploration site. By defining marginal exploration value (MEV), and using it as a proxy for revenue, much of the extensive body of revenue management control and optimization algorithms can potentially be used (see Chapter 3).

Given the logistics and exploration focus of this thesis, marginal exploration value was a logical value function to use. However, the framework developed is flexible enough to allow for the definition of arbitrary revenue proxies as long as they can be defined through processes, and fit within the 10 canonical classes of supply. One can imagine the implementation of other exploration-based value functions, or even functions that focus on risk management, basic logistic performance, etc.

\section{SpaceNet RM}

The third important contribution is the implementation of SpaceNet RM, which builds on SpaceNet's existing scenario definition, demand modeling, and simulation capabilities to implement the EMEV and bid-price algorithms developed in thesis.

\subsection{Recommendations}

The two variations of the Winnebegos and Jeeps discussed in Section highlight how optimal capacity allocation may significantly differ from the nominal in shorter missions, particularly if spares demand is a significant portion of overall demand. As an exploration architecture matures, the trend is for vehicle performance to fall short of expectations while demand exceeds expectations. The result of this is usually reduced exploration capability, often in the form of reduced mission durations. Another key factor in adjusting the optimal capacity allocation is the spares K-factor, which determines how quickly spares demands reach their long term nominal value (i.e. the deterministic case mean value). In these cases, it is important to recalculate capacity allocations to account for the changed demand profiles, rather than just scaling the deterministic (long duration) case.

As more dynamic mission types such as the Winnebegos and Jeeps concept become a reality, it is important to integrate probabilistic demand into the logistical design process as early as possible. These mission types tend to have reduced warehousing capacity and may have no true "steady state". Integrating revenue management into integrated modeling and simulation is one way to provide insight into the effect of various mission parameters at the early stages of mission concept development, allowing these insights to be fed back into the process. 


\subsection{Future Work}

Several areas of future work are being proposed as follow-on work to this research. These areas broadly fall into two categories: extension of the conceptual framework and improvements to SpaceNet. The key improvements to the conceptual framework should be made in the future:

- Capacity allocation for habitats and exploration items. As discussed in Chapter 4, the RM implementation in this thesis requires the user to specify the habitation and infrastructure items (CoS 8), as well as to provide exploration goals ( $\operatorname{CoS} 6)$. With this information, SpaceNet RM can then determine the demand of crew consumables (CoS 2 and 3), spares, and other classes of supply, and then allocate capacity accordingly. However, the framework would be even more powerful if it could make an integrated capacity allocation including spares, habitation, and exploration items.

- The ability to handle stationary surface outpost missions. These missions are difficult because the ability to store large amounts of safety stocks and pre-position large amounts of cargo makes pricing the different supply classes very difficult. In the current framework, the warehousing of large amounts of supplies drowns out the stochasticity in the demand model and essentially turns a scenario into the deterministic case. To some extent this mirrors real life, but future versions of SpaceNet RM should attempt to handle large warehousing. One potential solution is to discount supply items beyond a certain threshold, much like a net present value calculation discounts earnings in the far future more than earnings in the near future.

- Robotic agents. In order to account for this, exploration capability (EC) must be updated to include contributions from robotic agents. Since MEV is essentially a probabilistic derivative of EC, it would automatically be updated as well.

In addition to the conceptual framework updates, the following software architecture upgrades would be required:

- User-defined value functions. As discussed above, the framework developed here is flexible enough to allow for the definition of arbitrary revenue proxies as long as they can be defined by processes, and fit within the 10 canonical classes of supply. Enabling the user to input custom value functions would greatly broaden the relevance and value of SpaceNet RM in mission design and evaluation. A simple implementation might involve allowing the user to give weights to exploration value, risk, exploration mass delivered, etc.

- Detailed stowage and restraint. One of the limitations of the SpaceNet RM implementation is the ability to handle complex nested stowage and restraint models. In the examples shown in Chapter 5, a per-class tare factor was used as an estimate of the mass associated with containers, attachment fittings, and logistics modules required to transport cargo in space. However, a more precise stowage and accommodation model would allow for better modeling fidelity, and better align the SpaceNet RM model with the ISCMLA model. 


\section{Appendix A}

\section{Network Representation}

\section{A.1 The Static Network}

The static network's nodes represent various physical locations in space. Three types of nodes have been identified:

1. Surface nodes: nodes on the surface of a terrestial body. Examples include NASA Kennedy Space Center on Earth and Mare Tranquilitatis on the Moon.

2. Orbit nodes: nodes in the orbit of a body. Examples include Low Earth Orbit (LEO) or Low Lunar Orbit (LLO).

3. Lagrange: point locations in an orbital configuration where a small object affected only by gravity can theoretically be stationary. Examples include the five Earth Moon Lagrange Points.

The arcs represent the physical connections between two nodes, that is, the family of trajectories that can be traversed between two physical locations. Within arcs are trajectories which represent the actual paths between the two nodes in the time-space continuum.

\section{A.2 Time Expanded Network}

The TEN can be thought of as a sequence of "snapshots" of the static network. In each snapshot, the set of arcs and the feasible trajectories, i.e. the set of orbital paths, within the arcs are updated based on orbital dynamics. For an arc to exist in the TEN, it must:

- Exist in the static network

- Create a connection that moves forward in time (i.e. no time travel)

- Represent a feasible transfer

In practice, the set of arcs never changes from snapshot to snapshot in the TEN; rather the set of trajectories within the arcs changes to reflect the changes in energy required to traverse the arc.

By representing the space network in this time expanded form, the network definition is decoupled from the celestial mechanics that govern space travel, since the existence of the arc in the time expanded network assures that a feasible transfer between two nodes exists. In addition, the time expanded network allows for a series of decisions about space logistics to be made in a holistic framework. 
day 1

day 2

day 3

day 4

day 5

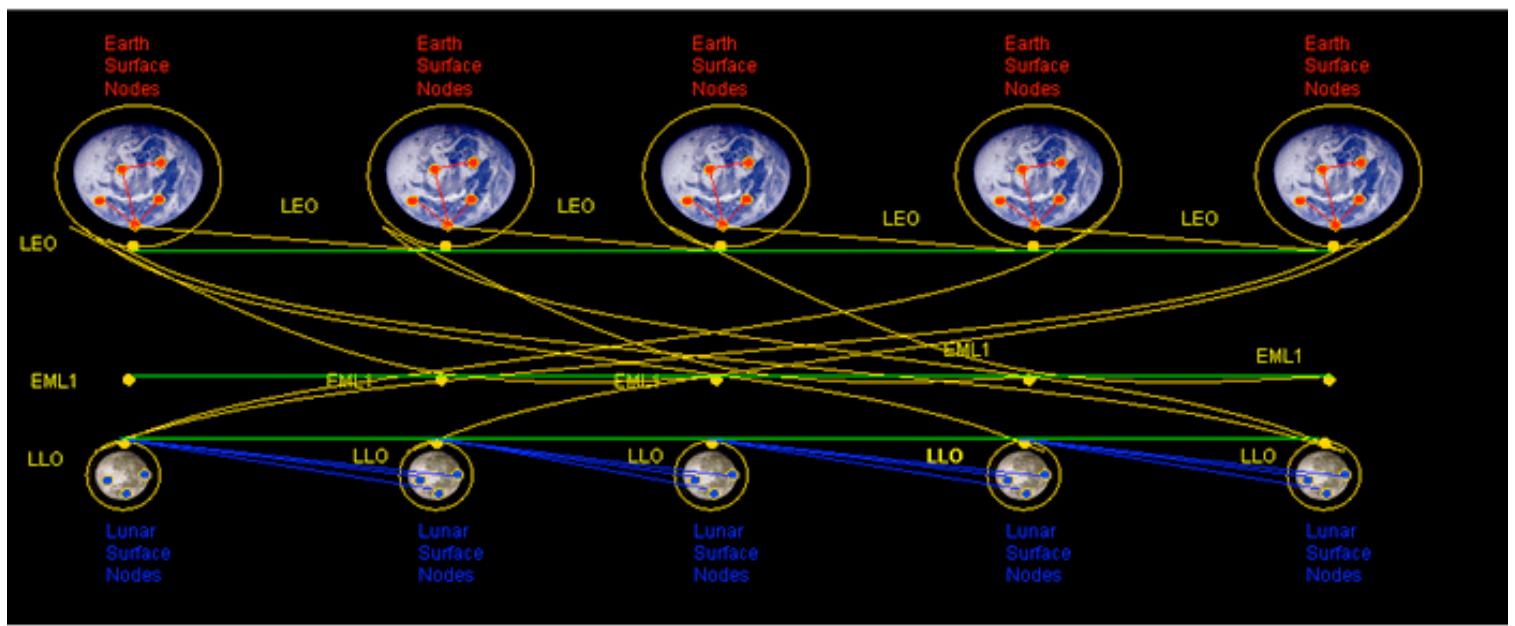

Figure A-1: An Earth-Moon time expanded network 


\section{Appendix B}

\section{Measures of Effectiveness}

\section{B.1 Basic Logistics Performance}

\section{B.1.1 Crew Surface Days (CSD) [crew-days]}

$C S D$ is the total cumulative number of crew surface days spent over over all $S$ nodes explored in a given scenario. It is specifically defined as:

$$
C S D_{t o t}=\Delta T \sum_{i=1}^{T} \sum_{j=1}^{S} N_{c r e w, i, j}
$$

$\Delta T=$ Earth days per SpaceNet time period

$T=$ total number of SpaceNet time periods in a scenario

$S=$ number of planetary exploration nodes visited in a scenario

$N_{\text {crew }, i, j}=$ number of crew present at exploration node $j$ during time period $i$

\section{B.1.2 Exploration Mass Delivered (EMD) [kg]}

EMD is the total amount of exploration mass delivered to all surface nodes over the course of a campaign (scenario). Exploration mass contains all items that are characterized as CoS 6 and CoS 8.

$$
E M D_{t o t}=\Delta T \sum_{i=1}^{T} \sum_{j=1}^{S}\left[\Delta m_{C O S 6, i, j}+\Delta m_{C O S 8, i, j}\right]
$$

$\Delta T=$ Earth days per SpaceNet time period

$T=$ total number of SpaceNet time periods in a scenario

$S=$ number of planetary exploration nodes visited in a scenario

$\Delta m_{C O S 6, i, j}=$ mass of exploration items delivered during time period $i$ to node $j$

$\Delta m_{C O S 8, i, j}=$ mass of infrastructure items delivered during time period $i$ to node $j$ 


\section{B.1.3 Total Launch Mass (TLM) $[\mathrm{kg}]$}

The total launch mass (including crew, elements, and all other CoS) lifted off from the surface of the Earth to accomplish a particular exploration scenario. SpaceNet calculates this metric as:

$$
T L M=\sum_{v=1}^{N_{f}} \sum_{e=1}^{E} m_{e m p t y, e} N_{e, v}+\sum_{k=0,8,9} m_{C o S, k}+\sum_{v=1}^{N_{f}} \sum_{e=1}^{E} m_{\text {crew }} N_{c r e w, e} N_{e, v}
$$

where

$N_{f}=$ total number of launches in a scenario

$E=$ number of element types in a scenario

$m_{\text {empty,e }}=$ empty mass ( $=$ dry (tare) mass + accommodation mass) of element type $e$

$N_{e, v}=$ number of type $e$ elements on launch $v$

$m_{C o S, k}=$ total mass launched for class of supply $k$ including CoS 1 (propellant), but excluding CoS

0,8 , and 9

$N_{\text {crew }, e}=$ number of crew present on type $e$ elements

$m=$ nominal mass of a crew member (usually taken to be $220 \mathrm{lbs}=100 \mathrm{~kg}$ )

TLM is integral to the calculation of other MOEs, particularly Relative Exploration Capability (REC). The total launch mass for Apollo 17 was approximately 2,930 metric tons, about 3 million kilograms.

\section{B.1.4 Up-mass Capacity Utilization (UCU) $[0,1]$}

A key metric to understand exploration logistics efficiency is that of cargo capacity utilization. Each propulsive and non-propulsive element (CoS 9) has a cargo capacity (in terms of mass and volume) associated with it. In some cases for propulsive elements such as the Earth Departure Stage (EDS), this capacity is zero, but for non-propulsive elements, the cargo capacity is usually non-zero. SpaceNet quantifies the fraction of available up-mass (launched from Earth) cargo capacity that is actually used for all classes-of-supply other than infrastructure ( $\mathrm{CoS} 8)$, transportation and carriers $(\mathrm{CoS} 9)$, propellant $(\operatorname{CoS} 1)$, and crew ( $\operatorname{CoS} 0)$. The upmass capacity utilization in a scenario is the ratio of the mass of tracked supply classes launched over all elements, divided by the total usable cargo mass capacity (= maximum cargo up-mass capacity less accommodation mass):

$$
U C U=\frac{\sum_{e=1}^{E I} \sum_{k=2-7,10} m_{C o S, k}}{\sum_{e=1}^{E I}\left(m_{c a p, e}-m_{\text {accom }, e}\right)}
$$

where

$E I=$ number of instantiated elements in a scenario

$m_{C o S, k, e}=$ total mass of class of supply $\mathrm{k}$ carried in instantiated element $e$ at time or reentry

$m_{c a p, e}=$ up-mass cargo capacity of instantiated element $e$

$m_{\text {accom }, e}=$ accommodation mass of instantiated element $e$

A completely efficient transportation system has a capacity utilization of unity, while a UCU of zero means that all elements are being launched empty. Various factors can result in capacity utilization below unity. These include volume-constrained payloads, packing inefficiencies, and parent element constraints (where the launch vehicle capability is the binding constraint, not the carriers cargo capacity). 


\section{B.1.5 Return-mass Capacity Utilization (RCU) $[0,1]$}

Another important metric to quantify is the fraction of available non-destructive return-mass (returned to Earth) cargo capacity that is actually used for all classes of supply other than infrastructure $(\operatorname{CoS} 8)$, transportation and carriers ( $\operatorname{CoS} 9)$, propellant $(\operatorname{CoS} 1)$, and crew $(\operatorname{CoS} 0)$. The non-destructive return-mass capacity utilization in a scenario is the ratio of the mass of tracked supply classes returned to Earth over all elements, divided by the total usable cargo return-mass capacity (= maximum cargo return-mass capacity less accommodation mass):

$$
R C U=\frac{\sum_{e=1}^{E I} \sum_{k=2-7,10} m_{C o S, k}}{\sum_{e=1}^{E I}\left(m_{\text {returncap }, e}^{r}-m_{\text {accom }, e}\right)}
$$

where

$E I=$ number of instantiated elements in a scenario

$m_{C o S, k, e}=$ total mass of class of supply $\mathrm{k}$ carried in instantiated element $e$ at Earth launch

$m_{\text {returncap }, e}=$ non-destructive return-mass cargo capacity of instantiated element $e$

$m_{\text {accom }, e}=$ accommodation mass of instantiated element $e$

Similarly to UCU, an RCU of zero means that all elements launch from and returning to the Earth are empty upon return, while a RCU of 1.0 indicates that the non-destructive return-mass cargo capacity of the system is being fully utilized. RCU reflects the benefits obtained by being able to return samples (CoS 603) or failed equipment back (CoS 703) for inspection on Earth.

\section{B.2 Exploration Capability}

\section{B.2.1 Exploration Capability [crew-day-kg]}

To first order, the exploration capability is the amount of time the crew gets to spend doing exploration and research at a surface node, multiplied by the amount of total exploration mass they have to do the job at each node visited during the scenario. The amount of time the crew can spend doing exploration and research while at a surface node is limited by a number of factors. These sources of crew non-availability include: housekeeping activities, maintenance and repair, in-situ crew activity planning/scheduling, medical, EVA preparation, and physiological (exercise, sleep/rest, eating). Exploration capability (EC) is defined as:

$$
E C=\Delta T \sum_{i=1}^{T}\left(1-\alpha_{i}\right) N_{c r e w, i}\left(m_{C O S 6, i}+m_{C O S 8, i}\right)
$$

In order to provide exploration capability, crew and exploration equipment have to be co-located at the same node concurrently. Crew at a surface node without exploration equipment does not produce benefit, and conversely exploration equipment at a node without crew to operate that equipment does not provide any benefit either. Note that generally the exploration capability is not the same as simply the product of total exploration mass delivered and total crew surface days in the entire scenario. However, when both crew and exploration mass always travel together, as during the Apollo missions, then EC can be simplified to:

$$
E C=E M D \times E C
$$




\section{B.2.2 Relative Exploration Capability}

The relative exploration capability is a normalized measure of exploration logistics efficiency. It is based on the well-established theory of linked index numbers, which are used to measure changes in economic productivity due to technology improvements. The exploration capability metric, Eq. (10), is explicitly a function of the number of crew and the exploration mass present at each node. It is also implicitly a function of all classes of supply since crew provisions, crew operations equipment, propellants, carriers, spares etc. are needed to produce crew at the surface node. A relative exploration capability metric needs to account for the necessary use and consumption of these resources as well in establishing overall logistics efficiency. REC is defined as

$$
R E C_{b, a}=\frac{E C_{t o t}^{b} / E C_{t o t}^{a}}{\prod_{k}\left(\frac{m_{C O S k}^{b}}{m_{C O S k}^{a}}\right)^{\beta_{k}}}
$$

where

$$
\beta_{k}=\frac{\omega_{k}^{a}+\omega_{k}^{b}}{2}
$$

given the following definitions:

$E C_{t o t}=$ exploration capability of a given scenario

$m_{C O S_{k}}=$ total mass of $C O S_{k}$

$\omega_{k}=$ mass fraction of $C O S_{k}$

SpaceNet uses Apollo 17 as the basis for normalization (i.e., REC $=1$ for Apollo 17), thus an interplanetary supply chain with an REC $>1$ would indicate a more efficient supply chain technology than Apollo 17 because more exploration capability is being provided for each unit of mass launched from Earth. Conversely an REC $<1$ would indicate a less efficient supply chain technology than Apollo 17. 


\section{Appendix C}

\section{ATHLETE}

The revolutionary ATHLETE (All-Terrain Hex-Legged Extra-Terrestrial Explorer) mobility platform, developed by JPL with support from Johnson and Ames Centers, Stanford University, and Boeing, is based on six wheels at the ends of six multi-degree-of-freedom limbs. Because each limb has enough degrees of freedom for use as a general-purpose leg, the wheels can be locked and used as feet to walk out of excessively soft or other extreme terrain. This allows wheels and actuators to be sized for nominal terrain, resulting in substantial mass savings. In addition, each limb has sufficient degrees-of-freedom to use as a general-purpose manipulator. As such, ATHLETE is capable of either autonomously performing or assisting in the loading, transportation, manipulation, and depositing payloads to essentially any desired sites of interest.

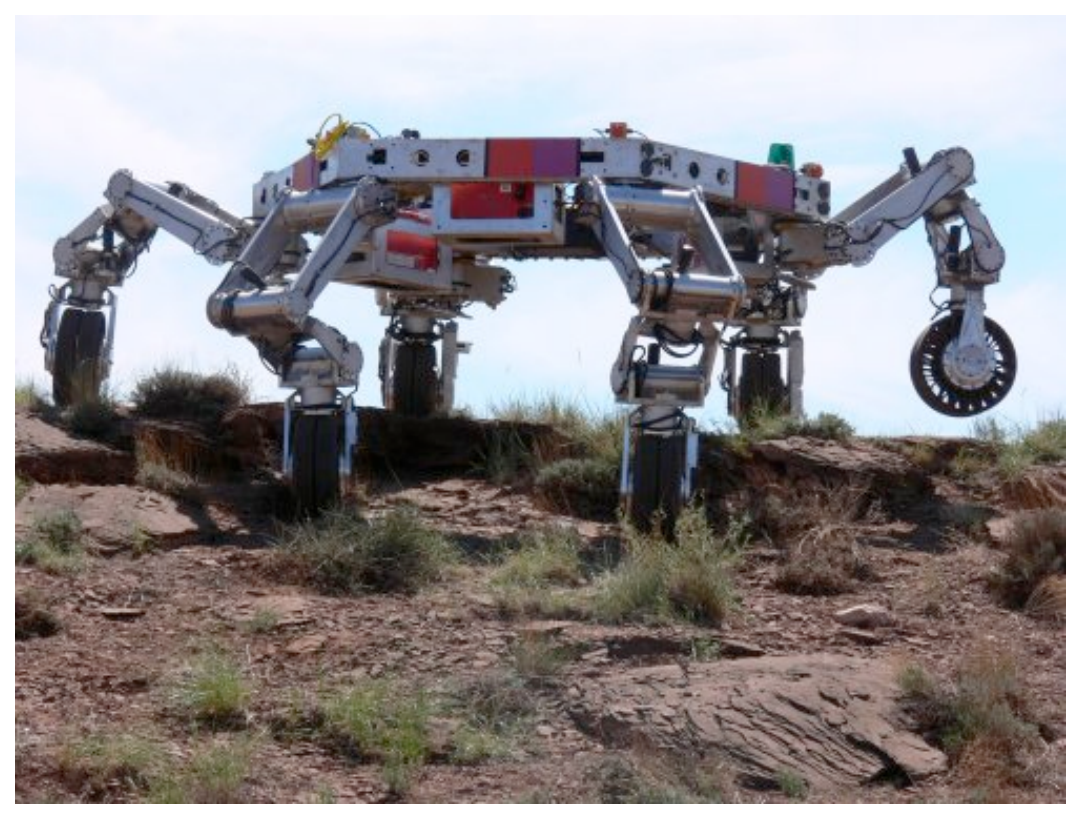

Figure C-1: All-Terrain Hex-Legged Extra-Terrestrial Explorer (ATHLETE) robotic vehicle 


\section{Bibliography}

[1] S. E. Bodily and L. R. Weatherford, "Perishable-asset Revenue Management: Generic and Multiple-price Yield Management with Diversion," Omega, vol. 23, no. 2, pp. 173-185, 1995.

[2] E. Gralla, S. Shull, O. de Weck, G. Lee, and R. Shishko, "A Modeling Framework for Interplanetary Supply Chains," AIAA 2006-7229, AIAA SPACE 2006 Conference and Exposition, San Jose, California, 19 - 21 September 2006.

[3] A. Siddiqi, O. de Weck, and S. A. Shull, "Matrix Methods Analysis of International Space Station Logistics," AIAA-2008-7605, AIAA SPACE 2008 Conference and Exposition, San Diego, California, September 2008.

[4] NASA, "Exploration Systems Architecture Study," NASA-TM-214062, NASA, 2005.

[5] S. A. Shull, Integrated Modeling and Simulation of Lunar Exploration. PhD thesis, Massachusetts Institute of Technology, May 2007.

[6] N. Armar, A. Siddiqi, O. de Weck, G. Lee, E. Jordan, and R. Shishko, "Design of Experiments in Campaign Logistics Analysis," AIAA-2008-7684, AIAA SPACE 2008 Conference and Exposition, San Diego, California, September 2008.

[7] M. Müller-Bungart, Revenue Management with Flexible Products, Models and Methods for the Broadcasting Industry. Springer, 2007.

[8] G. Lee, E. Jordan, R. Shishko, N. Armar, A. Siddiqi, and O. de Weck, "SpaceNet: Modeling and Simulating Space Logistics," AIAA-2008-7747, AIAA SPACE 2008 Conference and Exposition, San Diego, California, September 2008.

[9] A. M. Law, Simulation Modeling and Analysis. McGraw-Hill, 4th ed., 2007.

[10] K. T. Talluri and G. J. van Ryzin, The Theory and Practice of Revenue Management. New York: Springer, 2004.

[11] G. Gallego and R. Phillips, "Revenue Management of Flexible Products," Manufacturing Service Operations Management, vol. 6, no. 4, pp. 321-337, 2004.

[12] W.-C. Chiang, J. C. H. Chen, and X. Xu, "An Overview of Research on Revenue Management: Current Issues and Future Research," International Journal of Revenue Management, vol. 1, no. 1, pp. 97-128, 2007.

[13] T. Elliott, "Maximising Revenue Production While Cutting Costs: An Airline Industry Mandate," Journal of Revenue and Pricing Management, vol. 1, pp. 355-268, 2003. 
[14] E. A. Boyd, "Airline Alliance Revenue Management," OR/MS Today, vol. Vol. 25, pp. pp.28-31, 1998.

[15] K.-K. Lai and W.-L. Ng, "A Stochastic Approach to Hotel Revenue Optimization," Computers \& Operations Research, vol. 32, no. 5, pp. 1059-1072, 2005.

[16] M. Rothstein, "Hotel Overbooking as a Markovian Sequential Decision Process," Decision Sciences, Jan 1974.

[17] G. R. Bitran and T.-Y. Leong, Hotel Sales and Reservations Planning. Cambridge, Mass.: Sloan School of Management, Massachusetts Institute of Technology, 1989.

[18] R. Cross, Revenue Management: Hard-Core Tactics for Market Domination. London: Orion Books Ltd., 1997.

[19] J. Pachon, E. Iakovou, and I. Chi, "Vehicle Fleet Planning in the Car Rental Industry.," Journal of Revenue \& Pricing Management, vol. 5, no. 3, pp. 221-236, 2006.

[20] S. A. Maragos, Revenue Management for Ocean Carriers: Optimal Capacity Allocation with Multiple Nested Freight Rate Classes. PhD thesis, Massachusetts Institute of Technology, 1994.

[21] K. Coulter, "Decreasing Price Sensitivity Involving Physical Product Inventory: A Yield Management Application," Journal of Product and Brand Management, vol. 10, pp. 301-317, 2001.

[22] Y. Aviv and A. Pazgal, "A Partially Observed Markov Decision Process for Dynamic Pricing," Management Science, vol. 51, pp. 1400-1416, 2005.

[23] M. Hatwin, "The Practicalities and Benefits of Applying Revenue Management to Grocery Retailing, and the Need for Effective Business Rule Management," Journal of Revenue and Pricing Management, vol. 2, pp. 61-67, 2003.

[24] B. Lippman, "Retail Revenue Management - Competitive Strategy for Grocery Retailers," Journal of Revenue and Pricing Management, vol. 2, pp. 229-233, 2003.

[25] S. Nair and P. Bapna, "An Application of Yield Management for Internet Service Providers," Naval Research Logistics, vol. 48, pp. 348-362, 2001.

[26] G. Bitran and R. Caldentey, "An Overview of Pricing Models for Revenue Management," Manufacturing and Service Operations Management, vol. 5, pp. 203-230, 2003.

[27] B. Smith, J. Leimkuhler, R. Darrow, and J. Samuels, "Yield Management at American Airlines," Interfaces, vol. 22, pp. 8-31, 1992.

[28] M. Beckmann, "Decision and Team Problems in Airline Reservations," Econometrica, vol. 26, pp. $134-145,1958$.

[29] H. Thompson, "Statistical Problems in Airline Reservation Control," Operations Research Quarterly, vol. 12, pp. 167-185, 1961.

[30] R. Chatwin, "Continuous-Time Airline Overbooking with Time Dependent Fares and Refunds," Transportation Science, vol. 33, pp. 182-191, 1999. 
[31] M. Rothstein, "An Airline Overbooking Model," Transportation Science, vol. 5, pp. 180-192, 1971.

[32] J. Subramanian, S. Stidham, and C. Lautenbacher, "Airline Yield Management with Overbooking, Cancellations and No-Shows," Transportation Science, vol. 33, pp. 147-167, 1999.

[33] R. L. Phillips, Pricing and Revenue Optimization. Stanford University Press, 2005.

[34] K. Littlewood, "Forecasting and Control of Passenger Bookings," AGIFORS Symposia, vol. 12, 1972.

[35] G. van Ryzin and J. McGill, "Revenue Management Without Forecasting of Optimization: An Adaptive Algorithm for Determining Airline Seat Protection Levels," Management Science, vol. 46 , no. 6 , pp. $760-775,2000$.

[36] A. Gosavi, N. Bandla, and T. K. Das, "A Reinforcement Learning Approach to a Single Leg Airline Revenue Management Problem with Multiple Fare Classes and Overbooking," IIE Transactions, vol. 34, pp. 729-742, 2002.

[37] D. Bertsimas and I. Popescu, "Revenue Management in a Dynamic Network Environment," Transportation Science, vol. 37, no. 3, pp. 257-277, 2003.

[38] A. Möller, W. Romisch, and K. Weber, "A New Approach to O-D Revenue Management Based on Scenario Trees," Journal of Revenue and Pricing Management, vol. 3, no. 3, pp. 265-276, 2004 .

[39] A. Bhatia and S. Parekh, "Optimal Allocation of Seats by Fare." Presentation by Trans World Airlines to AGIFORS Reservations Study Group, 1973.

[40] H. Richter, "The Differential Revenue Method to Determine Optimal Seat Allocations by Fare Type," AGIFORS Symposium Proceedings, vol. 22, pp. 339-362, 1982.

[41] B. Titze and R. Griesshaber, "Realistic Passenger Booking Behaviour and the Simple Low-Fare/high fre seat allotment model," Proceedings of the AGIFORS symposium., http://worldcat.org.

[42] M. Mayer, "Seat Allocation, or a Simple Model of Seat Allocation Via Sophisticated Ones," , AGIFORS Symposium, Key Biscane, Florida, 1976.

[43] P. P. Belobaba, "Airline Yield Management : An Overview of Seat Inventory Control," Transportation science., vol. 21, no. 2, http://worldcat.org.

[44] P. P. Belobaba, Air Travel Demand and Airline Seat Inventory Management. PhD thesis, Massachusetts Institute of Technology, Cambridge, MA, http://worldcat.org.

[45] P. P. Belobaba, "Optimal vs. Heuristic Methods for Nested Seat Allocation," in Proceedings of the AGIFORS Reservations and Yield Management Study Group, (Brussels), pp. 28-53, 1992.

[46] S. L. Brumelle and J. I. McGill., "Airline Seat Allocation with Multiple Nested Fare Classes," Operations Research, vol. 41, pp. 127-137, 1993.

[47] J. Papastavrou, S. Rajagopalan, and A. Kleywegt, "The Dynamic and Stochastic Knapsack Problem with Deadlines," Management Science, Dec 1996. 
[48] A. J. Kleywegt and J. D. Papastvrou, "The Dynamic and Stochastic Knapsack Problem," Operations Research, vol. 46, pp. 17-35, 1998.

[49] A. Kleywegt and J. Papastavrou, "The Dynamic and Stochastic Knapsack Problem with Random Sized Items," Operations Research, vol. 49, pp. 26-41, 2001.

[50] K. Talluri and G. V. Ryzin, "An Analysis of Bid-Price Controls for Network Revenue Management," Management Science, vol. 44, no. 11, pp. 1577-1593, 1998.

[51] B. Blanchard, Systems Engineering and Analysis. Upper Saddle River, NJ: Prentice Hall, third edition ed., 1998.

[52] NASA, "Maintainability Program Management Considerations," 1994.

[53] G. Lee, E. Jordan, R. Shishko, E. Gralla, S. Shull, A. Siddiqi, and O. de Weck, "SpaceNet 1.3 User Manual," , MIT Strategic Engineering Group, 2006.

[54] T. C. Bachman and R. C. Kline, "Model for Estimating Spare Parts Requirements for Future Missions," AIAA 2004-5978, AIAA SPACE 2004 Conference and Exposition, Oct 2004.

[55] C. Hulten, "Divisia Index Numbers," Econometrica, vol. 41, Nov 1979.

[56] J. A. Rice, Mathematical Statistics and Data Analysis. Duxbury Press, 1995.

[57] A. H. G. Rinnooy Kan, L. Stougie, and C. Vercellis, "A Class of Generalized Greedy Algorithms for the Multi-knapsack Problem," Discrete Applied Mathematics, vol. 42, no. 2-3, pp. 279-290, 1993.

[58] B. H. Wilcox, "ATHLETE: An Option for Mobile Lunar Landers," in IEEE Aerospace Conference 2008, pp. 1-8, 2008.

[59] W. J. Larson and L. K. Pranke, Human Spaceflight: Mission Analysis and Design. McGraw-Hill, 2000. 